\title{
Successful user operation of a superconducting radio-frequency photoelectron gun with $\mathrm{Mg}$ cathodes
}

\author{
J. Teichert๑, ${ }^{1,}$ A. Arnold $\odot,{ }^{1}$ G. Ciovati, ${ }^{2}$ J.-C. Deinert $\odot,{ }^{1}$ P. Evtushenko $\odot,{ }^{1}$ M. Justus,${ }^{1}$ \\ J. M. Klopf $\odot,{ }^{1}$ P. Kneisel, ${ }^{2}$ S. Kovalev $\odot,{ }^{1}$ M. Kuntzsch $\odot,{ }^{1}$ U. Lehnert, ${ }^{1}$ P. Lu, ${ }^{1, \dagger}$ S. Ma $\odot,{ }^{1}$ \\ P. Murcek, ${ }^{1}$ P. Michel $\odot,{ }^{1}$ A. Ryzhov $\odot,{ }^{1}$ J. Schaber $\odot,{ }^{1,3}$ C. Schneider, ${ }^{1}$ R. Schurig, ${ }^{1}$ \\ R. Steinbrück, ${ }^{1}$ H. Vennekate $\odot,{ }^{1, \$}$ I. Will, ${ }^{4}$ and R. Xiang ${ }^{1}$ \\ ${ }^{1}$ Helmholtz-Zentrum Dresden-Rossendorf, 01328 Dresden, Germany \\ ${ }^{2}$ Thomas Jefferson National Accelerator Facility, Newport News, Virginia 23606, USA \\ ${ }^{3}$ TU Dresden, 01062 Dresden, Germany \\ ${ }^{4}$ Max-Born-Institut, 12489 Berlin, Germany
}

(Received 14 November 2020; accepted 1 February 2021; published 4 March 2021)

\begin{abstract}
At the electron linac for beams with high brilliance and low emittance (ELBE) center for high-power radiation sources, the second version of a superconducting radio-frequency (SRF) photoinjector has been put into operation and has been routinely applied for user operation at the ELBE electron accelerator. SRF guns are suitable for generating a continuous wave electron beam with high average currents and high beam brightness. The SRF gun at ELBE has the goal to generate short electron pulses with bunch charges of 200-300 pC at typical repetition rates of $100 \mathrm{kHz}$ for the production of superradiant, coherent terahertz radiation. The SRF gun includes a $3.5-$ cell, $1.3-\mathrm{GHz}$ niobium cavity and a superconducting solenoid. A support system with liquid nitrogen $\left(\mathrm{LN}_{2}\right)$ cooling allows the operation of normal-conducting, high quantum efficiency photocathodes. We present the design and performance of the SRF gun as well as beam measurement results of the operation with $\mathrm{Mg}$ photocathodes at an acceleration gradient of $8 \mathrm{MV} / \mathrm{m}$ (4 MeV kinetic energy). In the last section, we discuss the SRF gun application for production of coherent terahertz radiation at the ELBE facility.
\end{abstract}

DOI: 10.1103/PhysRevAccelBeams.24.033401

\section{INTRODUCTION}

The "electron linac for beams with high brilliance and low emittance (ELBE)" is a user facility open for international research projects with electrons and secondary radiation. Located at the Helmholtz-Zentrum DresdenRossendorf (HZDR), the ELBE accelerator with its superconducting linac has the unique feature to operate in continuous wave (cw) mode for high average power output [1]. And quite obviously, a reliable cw electron source is required for this. There are various candidates of such $\mathrm{cw}$ sources, for instance, the state-of-the-art dc guns [2,3] or the conceptual relatively new superconducting radiofrequency (SRF) guns [4] as well as low-frequency normal conducting guns [5]. For the ELBE facility, a thermionic dc gun of $235 \mathrm{kV}$ routinely provides up to $77 \mathrm{pC}$ at $13 \mathrm{MHz}$

\footnotetext{
*j.teichert@hzdr.de

Present address: KLA-Tencor, Shanghai, China.

Present address: TJNAF, Newport News, Virginia.
}

Published by the American Physical Society under the terms of the Creative Commons Attribution 4.0 International license. Further distribution of this work must maintain attribution to the author(s) and the published article's title, journal citation, and DOI. for the high-power IR free-electron lasers (FELs) and other users. However, high bunch charge operation is highly demanded by several new attractive applications. For example, neutron time-of-flight experiments [6] require high bunch charge to improve the signal-to-noise ratio. High-power terahertz experiments [7] and Thomson backscattering experiments [8] need high bunch charge and short electron pulses to increase the intensity of the secondary radiation.

As part of the R\&D effort to explore a new cw injector for the ELBE center, the first SRF gun was developed and operated from 2007 to 2014 as the second injector [9]. This gun consisted of a 1.3-GHz 3.5-cell niobium cavity, a choke filter with a normal conducting photocathode, a drive laser, a beam transport system, and all other accessories. In April 2013, first lasing of one of the IR FELs at ELBE was demonstrated using the electron beam from this gun [10]. It represented an excellent demonstration that L-band SRF guns can work in a user facility, but significant improvement was needed for better gun performance.

Based on the experience of the first gun, an updated version has been installed in the accelerator hall since May 2014. This so-called SRF gun II is now able to achieve an acceleration gradient of $8 \mathrm{MV} / \mathrm{m}$, which corresponds to 20.5 MV/m peak field on axis. In combination with $\mathrm{Mg}$ 
cathodes and a 258-nm laser, this new gun routinely provides electron beams with bunch charge of up to $300 \mathrm{pC}$. This value is limited by the space charge effect on cathode surface. In past years, further improvements and optimization of components as well as operational experiences have increased the performance and reliability so that SRF gun II is now applied for user operation. In this paper, we introduce the main features of the gun and share the experience gained during the commissioning and operation for high-power terahertz user beam application.

The advantages of the ELBE SRF gun II are the high field gradient, the high duty factor beam operation due to its superconducting technology, and the good vacuum conditions suitable for sensitive photocathodes. Depending on the photocathode and the drive laser system, the repetition rate can be theoretically as high as gigahertz level. Thus, the concept of SRF guns is promising to achieve high gradient and high output power [11]. SRF guns are also suitable to be used in other applications, such as energy recovery linac projects [12], high duty factor x-ray FELs [13], and $\mathrm{MeV}$ ultrafast electron diffraction and microscopy [14].

\section{OVERVIEW OF SRF GUN II DESIGN}

The design concept of SRF gun II is similar to that of the previous gun version which is described in detail elsewhere [9]. But the cryomodule including cavity and superconducting solenoid has been rebuilt completely. In 2014, the old gun was replaced, whereas the cathode transfer system, electron beam lines, and diagnostics were refurbished and just slightly improved. Over the years, major changes were made at the UV drive laser and the fundamental power radio-frequency (rf) system. A summary of the main gun parameters is given in Table I. The following detailed discussions are focused on design issues that are not

TABLE I. SRF gun II main parameters.

\begin{tabular}{lcc}
\hline \hline & \multicolumn{2}{c}{ Value } \\
\cline { 2 - 3 } Parameter & Gun I & Gun II \\
\hline Type of cavity & \multicolumn{2}{c}{$1.3 \mathrm{GHz}$} \\
Frequency & \multicolumn{2}{c}{$\mathrm{cw}$} \\
Gun operation mode & $3.0 \mathrm{MeV}$ & $4.0 \mathrm{MeV}$ \\
Beam energy at gun exit & $6 \mathrm{MV} / \mathrm{m}$ & $8 \mathrm{MV} / \mathrm{m}$ \\
Acceleration gradient $E_{\text {acc }}$ & $16.2 \mathrm{MV} / \mathrm{m}$ & $20.5 \mathrm{MV} / \mathrm{m}$ \\
Peak field on axis & $7 \mathrm{MV} / \mathrm{m}$ & $14.5 \mathrm{MV} / \mathrm{m}$ \\
Cathode field & \multicolumn{2}{c}{$-5 \mathrm{kV}$} \\
dc bias at cathode & \multicolumn{2}{c}{$2 \mathrm{~K}$} \\
Liquid He temperature & \multicolumn{2}{c}{$258 \mathrm{~nm}$} \\
Dynamic He load at max $E_{\text {acc }}$ & $\mathrm{Cs}_{2} \mathrm{Te}$ & $\mathrm{Mg}$ \\
Drive laser wave length & $1 \%$ & $0.1 \%-0.3 \%$ \\
Photocathodes & \multicolumn{2}{c}{ Quantum efficiency }
\end{tabular}

discussed in previous papers and on subsystems that differ significantly in SRF gun II.

\section{A. Cryomodule design, cavity, SC solenoid, and cathode cooling}

The cryomodule is designed for $\mathrm{cw}$ operation of a superconducting $\mathrm{Nb}$ cavity cooled down to $2 \mathrm{~K}$. For this purpose, the cavity is housed in a titanium vessel filled with superfluid helium using a two-phase helium pipe that at the same time collects the evaporated gas to return it to the helium plant. The maximum heat load into the helium bath handled by this pipe can be as high as $40 \mathrm{~W}$ (or $2 \mathrm{~g} / \mathrm{s}$ ). This value is composed of $7 \mathrm{~W}$ static loss caused by the finite thermal isolation and up to $33 \mathrm{~W}$ dynamic loss. Since the latter is determined by the field inside the cavity, an electric heater is used to operate the cryomodule at constant helium heat load (or mass flow) regardless of the cavity gradient. The heater is placed in a small helium pot that serves as an additional helium reservoir and guarantees a constant fill level in the cryomodule. This has been proven advantageous to achieve a pressure stability of down to $0.1 \mathrm{mbar}$ rms in the whole helium system. A computer-aided design (CAD) layout of the cryomodule is shown in Fig. 1. The stainless steel vacuum vessel has a cylindrical shape with $0.7 \mathrm{~m}$ diameter and $1.3 \mathrm{~m}$ length. Both the He port and the $\mathrm{LN}_{2}$ port are on top of the cryomodule. The cryomodule is equipped with a liquid-nitrogen-cooled thermal shield operated at $77 \mathrm{~K}, 10-20$ layers of superinsulation foil, and a warm magnetic shield (close to room temperature) made of MUMETALL®.

For the high bunch charge operation, the magnetic lens was moved inside the cryomodule and is now located at about $0.7 \mathrm{~m}$ from the cathode. In contrast to SRF gun I, where a normal conducting solenoid was placed outside the module at about $1.1 \mathrm{~m}$, the new superconducting solenoid enables the compensation of space charge force earlier and, thus, improves the transverse emittance [15].

As shown in Fig. 1, the 1.3-GHz cavity consists of three cells with shapes like the design for the TeV-Energy Superconducting Linear Accelerator (TESLA) and a length-optimized half-cell. A fifth superconducting cell, called the choke filter, is surrounding the cathode area and prevents rf leakage into the cathode support system. Compared to SRF gun I, the half-cell is improved by a stiffening structure to reduce Lorentz force detuning [16]. Furthermore, the half-cell is now tuned to $80 \%$ of the onaxis peak field of the TESLA cells of the cavity, whereas this value was $60 \%$ in SRF gun I. This is possible by a permanent pretension at the half-cell tuner of the doubletuning system of the gun [9]. Together with the higher acceleration gradient of SRF gun II, it doubles the electric peak field at the cathode to $14.5 \mathrm{MV} / \mathrm{m}$ with respect to SRF gun I. This value is true if the cathode is retracted by $1.3 \mathrm{~mm}$ together with a bias of $-5 \mathrm{kV}$, as it turned out to be the optimum case for operation with $\mathrm{Mg}$ photocathodes and 


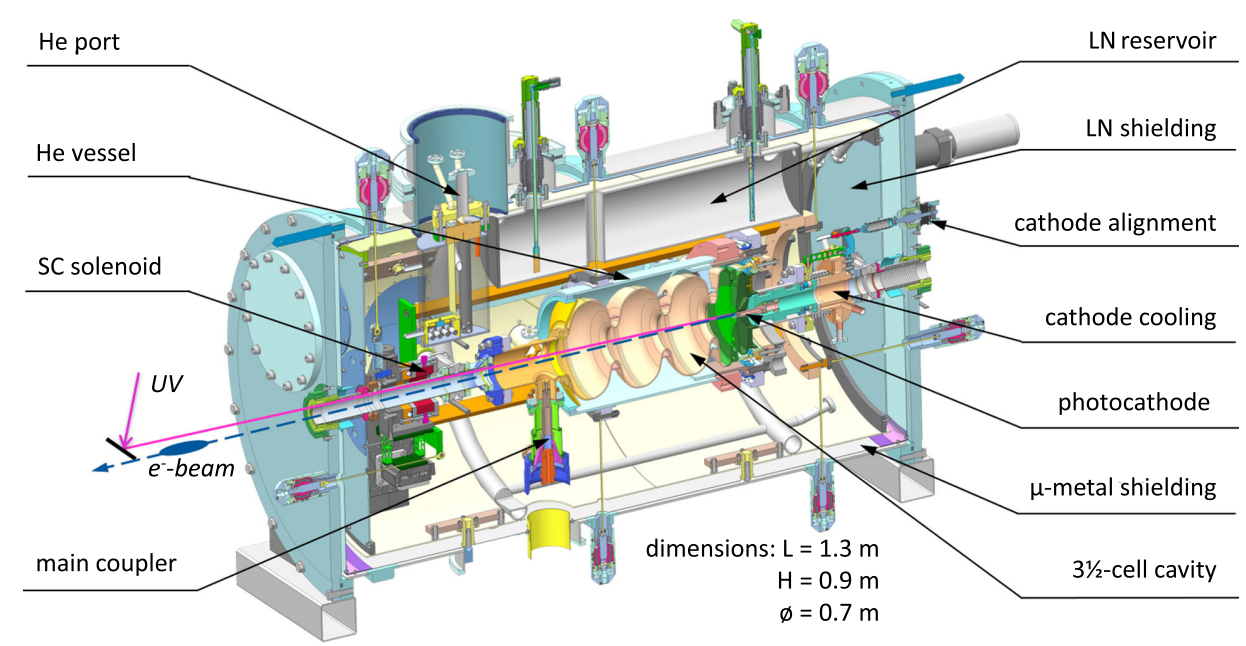

FIG. 1. Cross section of the ELBE SRF gun II cryomodule, holding a 3.5-cell gun cavity and a superconducting solenoid at about $70 \mathrm{~cm}$ from the cathode.

higher bunch charge $(200 \mathrm{pC})$ required for user operation at ELBE.

Within a collaboration project, the SRF gun II cavity was fabricated, treated, and tested at the Thomas Jefferson National Accelerator Facility (TJNAF) [17]. The cavity was vertically measured after final treatment at TJNAF in 2013 and later in the gun cryomodule with a copper cathode during the commission process in 2014. Since then, the performance of cavity has been monitored regularly. Some selected results are shown in Fig. 2. Compared to the last vertical test, the cavity lost about $30 \%$ of its performance because of increased field emission and degraded again after the first introduction of a $\mathrm{Cs}_{2} \mathrm{Te}$ cathode [18].

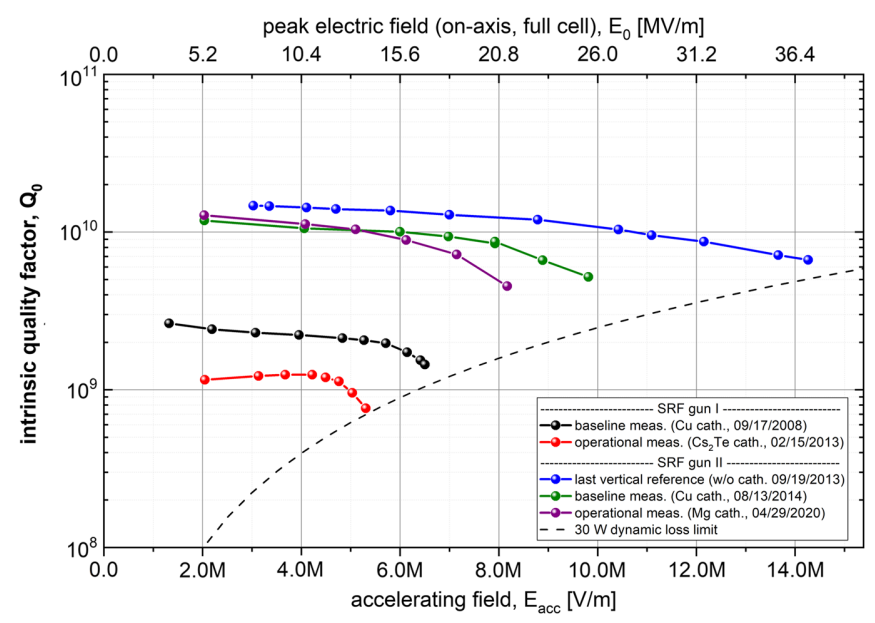

FIG. 2. Comparison of the intrinsic quality factor $Q_{0}$ as a function of the accelerating gradient $E_{\text {acc }}$ of SRF gun II. The diagram shows a significant improvement compared to SRF gun I, even though not all of the earlier vertical performance could be transferred into routine operation.
Nevertheless, the remaining gradient is still higher than that of SRF gun I. Over the past 5 years of operation, the cavity performance has remained constant despite many $\mathrm{Cs}_{2} \mathrm{Te}$ and $\mathrm{Mg}$ cathodes that have been used in the gun.

SRF gun II is equipped with the same double-tuning system as its predecessor. Thus, the half-cell can be tuned separately from the three TESLA cells [9]. Both tuners have been characterized at the cold state, where they show an excellent frequency resolution of $0.3 \mathrm{~Hz}$ per step (halfcell) and $0.8 \mathrm{~Hz}$ per step (TESLA cells) as well as negligible hysteresis. At the same time, the system provides a wide tuning range of almost $\pm 300 \mathrm{kHz}$ to compensate for various mechanical tolerances [19].

Another three main characteristics of the cavity are microphonics, Lorentz force detuning, and helium pressure sensitivity. Despite all of the disturbances, the total phase noise measured using a Rhode \& Schwarz FSWP phase noise analyzer from $10 \mathrm{~Hz}$ to $10 \mathrm{MHz}$ is $\sigma_{\varphi}=0.01^{\circ}$ at $1.3 \mathrm{GHz}$ or $\sigma_{\varphi}=23 \mathrm{fs}$ rms, respectively, and, thus, clearly fulfills our requirements. Lorentz force detuning leads to an overall frequency shift of $650 \mathrm{~Hz}$ for the typical operating gradient of $E_{\text {acc }}=8 \mathrm{MV} / \mathrm{m}$. The helium pressure sensitivity of $150 \mathrm{~Hz} / \mathrm{mbar}$ is almost exactly the same as for gun I.

The photocathode itself is thermally and electrically isolated from the cavity and cooled down with liquid nitrogen (Fig. 3). That allows the application of normal conducting photocathodes in the superconducting environment of the gun. The cathode cooling and support unit was designed for an easy exchange of the cathodes in the cold gun as well as for precise positioning. Therefore, three rotary feedthroughs at the back side of the module allow for axial cathode movement $( \pm 0.6 \mathrm{~mm})$ with respect to the cavity for best rf focusing but also for radial adjustment of the cathode in the half-cell. Additionally, a dc bias can be applied to the cathode in order to suppress multipacting. 


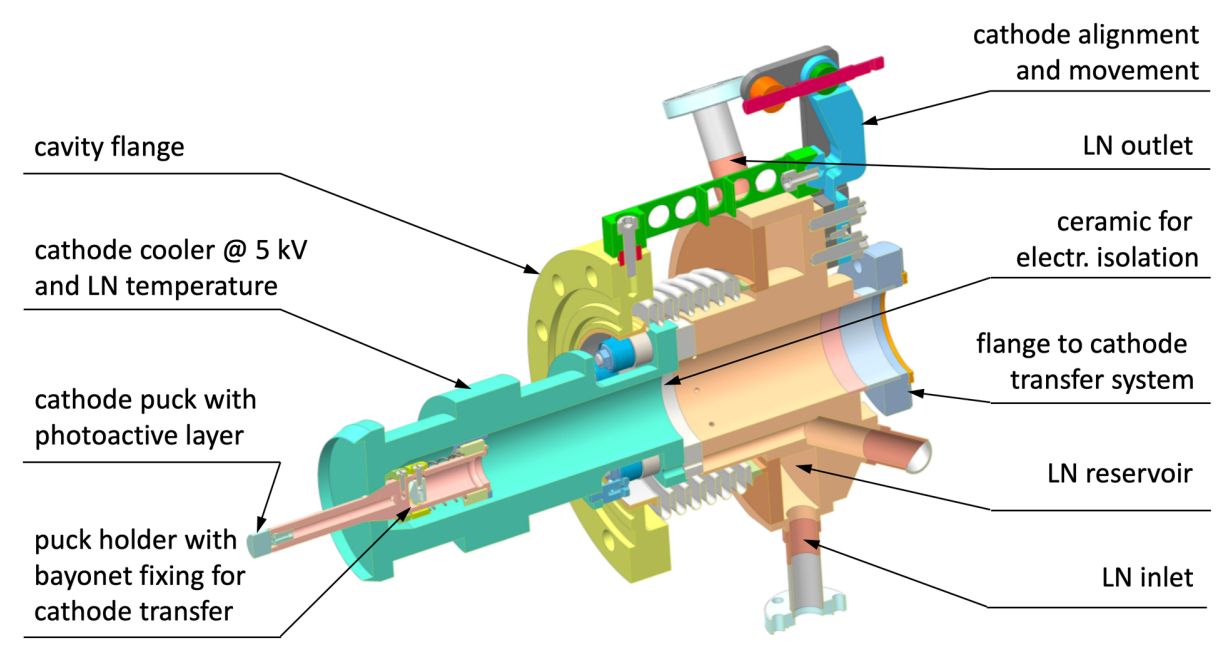

FIG. 3. Cathode holder and $\mathrm{LN}_{2}$ cooling system.

Multipacting was one of the main problems for the SRF gun I. As predicted by simulations, the circular gap between the cathode and cavity was prone to multipacting. Beside this geometrical reason, the contamination of the photocathode with cesium atoms arising from the $\mathrm{Cs}_{2} \mathrm{Te}$ deposition process intensified the problem. In cooperation with the universities in Rostock and in Siegen [20], extensive studies were carried out to mitigate multipacting by additional surface layers or surface microstructuring. For the SRF gun II with $\mathrm{Mg}$ cathodes, no special precautions are needed, as multipacting does not appear. In addition, even dark current typically on the order of $40 \mathrm{nA}$ at $8 \mathrm{MV} / \mathrm{m}$ has no negative effect on the terahertz user operation, so it is not discussed in this paper. However, a reference is given here in which the dark current issue is discussed in further detail [21].

The cavity is completed by a coaxial fundamental mode power coupler (FPC) and two TESLA-type higher-order mode (HOM) dampers. Thermal anchors made of copper braids are used to improve the heat conduction between HOM couplers and the helium vessel. Furthermore, the sapphire if feedthroughs of the HOM couplers can achieve a higher thermal conductivity between the inner and outer conductor of the coaxial line. Both reduce the thermal drift during gradient changes and improve cw capability of the cavity substantially. The FPC was developed at HZDR and couples rf power via an open end (the antenna tip) into the first cell of the cavity. The coupling factor (or external Q) is fixed in the range $6 \times 10^{6}<Q_{\text {ext }}<3 \times 10^{7}$. The cold part of the coupler is composed by a coaxial waveguide whose inner and outer conductors are connected to a conical ceramic cooled with liquid nitrogen. The warm parts of the coupler realize the transition between the coaxial and the rectangular waveguide used for low-loss rf transport. A second warm window made of quartz glass is located in the waveguide and acts as a vacuum barrier between the coupler vacuum and atmosphere.

\section{B. rf system and synchronization to drive laser}

An amplitude and phase stable rf field for acceleration as well as a low noise and low jitter locking of the photocathode laser to the rf reference are crucial for the stability and quality of the generated electron beam. In particular, for superconducting niobium cavities with their frequency detuning dominated by microphonics, the former must be realized by active feedback loops. For this purpose, two analog proportional control loops for amplitude and phase are combined into one controller (see Fig. 4).

Hence, the resonator is driven at exactly $1.3 \mathrm{GHz}$ (generator-driven resonator), and both amplitude and phase are continuously adjusted by two actuators (voltage-controlled phase shifter and attenuator). The transient signal is taken by a cavity field probe and determined with respect to amplitude (level detector) and phase (mixer). After low-pass filtering, both are compared with the target values provided by the programmable logic controller and supervisory control and data acquisition system of the accelerator. The differential voltages determined in this way control both actuators mentioned before. The stabilized rf signal is then amplified by a solid state power amplifier (SSPA) and brought to the cavity using a WR650 waveguide. To protect the amplifier, a rf isolator is used to dump the rf power that is reflected by the cavity into a rf load. Additionally, there is a possibility to adjust the cavity bandwidth by means of a three-stub waveguide tuner between 50 and $300 \mathrm{~Hz}$. The short-term amplitude stability of the amplitude control loop can reach $2 \times 10^{-4} \mathrm{rms}$ and the phase stability $0.01^{\circ} \mathrm{rms}$ (measured between $10 \mathrm{~Hz}$ and $10 \mathrm{MHz}$ ).

The required $\mathrm{rf}$ power is provided by a state-of-the-art SSPA from Sigmaphi Electronics. The 1300-MHz amplifier is composed by ten rf modules that are combined by a WR650 waveguide to reach up to $15 \mathrm{~kW} \mathrm{cw}$ with a bandwidth of $\pm 5 \mathrm{MHz}$. Each of the rf modules delivers 


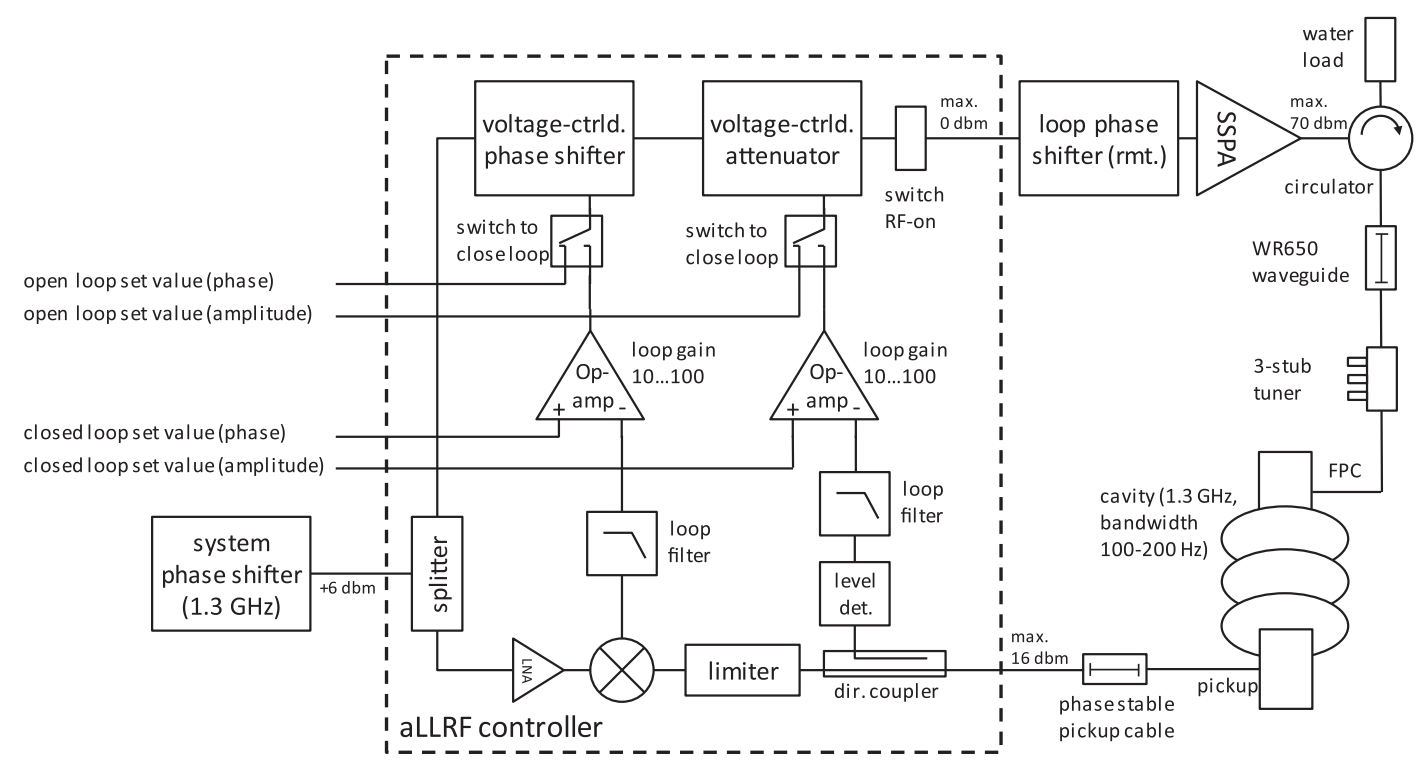

FIG. 4. Block diagram of the analog low-level rf controller.

$2 \mathrm{~kW}$ from four 500-W laterally-diffused metal-oxide semiconductor (LDMOS) transistors organized around a four-way combiner and cooled by a copper plate. The guaranteed minimum small signal gain is $73 \mathrm{~dB}$, whereas the expected efficiency at nominal rf power is $40 \%-45 \%$. The phase drift over an output power range of $2-15 \mathrm{~kW}$ is less than $8^{\circ}$. Gain linearity over the same dynamic range is less than $\pm 0.5 \mathrm{~dB}$. The rise and fall time is shorter than $100 \mathrm{~ns}$.

The synchronization of the four linac cavities of ELBE, the SRF gun, and the photocathode laser is realized using the common master oscillator of $13 \mathrm{MHz}$ and $1.3 \mathrm{GHz}$ (see Fig. 5). These frequencies are generated together with other frequencies required for operation by the RALAB accelerator master clock from LAURIN AG. The $1.3-\mathrm{GHz}$ reference signal is divided by an active rf splitter onto the low-level rf (LLRF) controllers of the harmonic buncher, the four ELBE linac cavities, and the SRF gun. Each of the latter two has a phase shifter along its signal path to adjust the exact timing between the accelerator and gun. A third phase shifter is used to change the timing of the laser with respect to gun rf. In addition to the already-

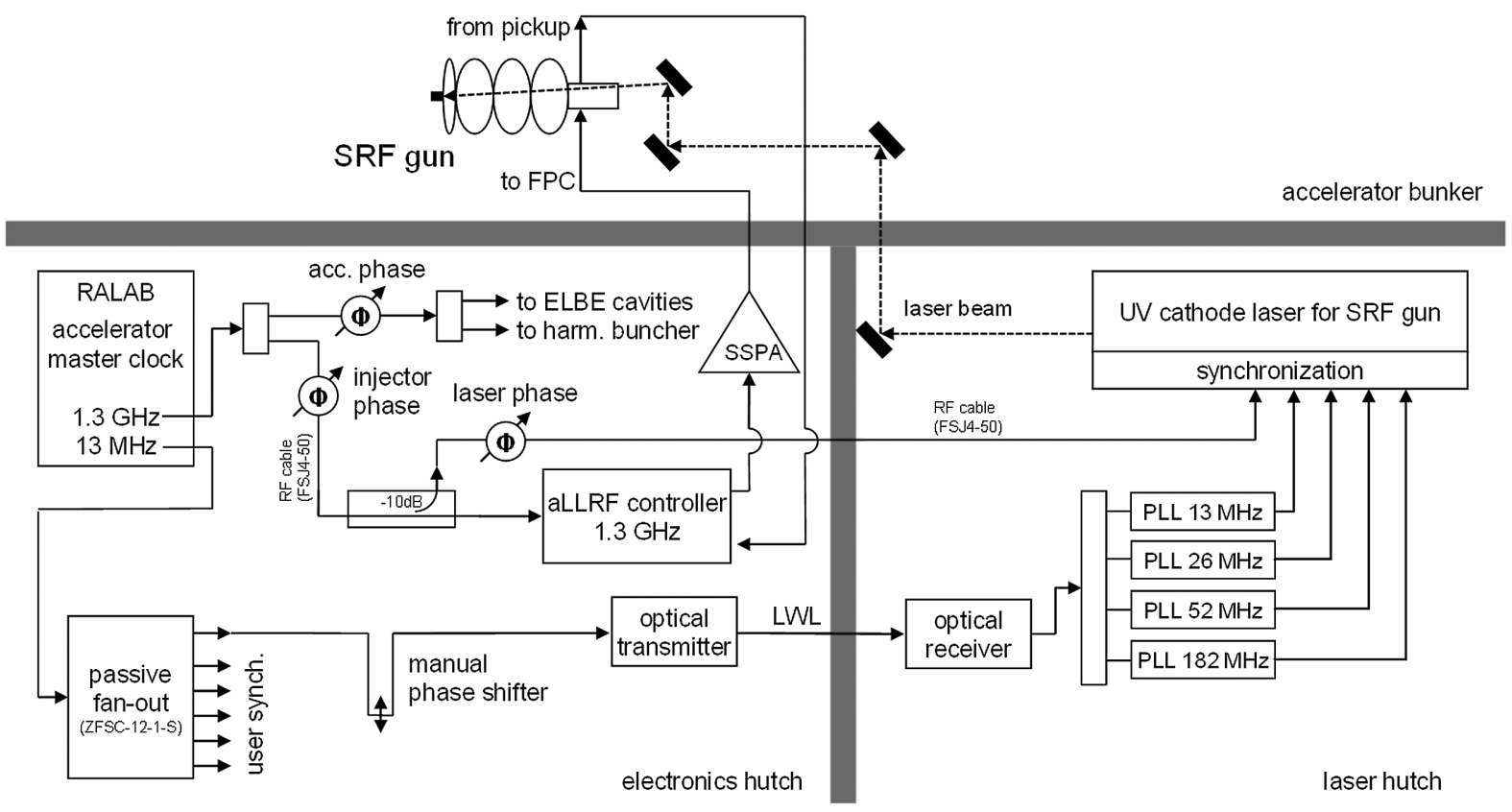

FIG. 5. Block diagram of rf system and laser synchronization to the ELBE master clock. 
TABLE II. Rms timing jitter of rf reference, SRF gun rf field, and laser oscillator measured using Rhode \& Schwarz FSWP phase noise analyzer.

\begin{tabular}{lccc}
\hline \hline Range & $\begin{array}{c}1.3 \mathrm{GHz} \\
\text { reference }\end{array}$ & SRF gun rf & $\begin{array}{c}\text { Laser } \\
\text { oscillator }\end{array}$ \\
\hline $1-10 \mathrm{~Hz}$ & $75.2 \mathrm{fs}$ & $101.3 \mathrm{fs}$ & $160.6 \mathrm{fs}$ \\
$10 \mathrm{~Hz}-10 \mathrm{MHz}$ & $27.3 \mathrm{fs}$ & $23.1 \mathrm{fs}$ & $85.7 \mathrm{fs}$ \\
$1 \mathrm{~Hz}-10 \mathrm{MHz}$ & $80.0 \mathrm{fs}$ & $103.9 \mathrm{fs}$ & $182.1 \mathrm{fs}$ \\
\hline \hline
\end{tabular}

mentioned high-frequency reference, the laser gets three further multiples of $13 \mathrm{MHz}$ provided by an optical transmission line and phase lock loops (PLLs) for 26, 52 , and $182 \mathrm{MHz}$. This guarantees a synchronous locking of the laser oscillator to the 13-MHz master clock, which is important for user experiments that use the same clock as well. The time jitter achieved with this setup is summarized for the 1.3-GHz reference, the SRF gun rf, and the laser oscillator in Table II.

\section{Mg photocathodes}

It is well known that the quality of the photocathodes is critical for the stability and reliability of photoinjector operation. In rf photoinjectors, the survivability in rf fields is another major issue, and, therefore, metal photocathodes are most suitable. Combined with a UV drive laser, a proper material is magnesium, which has a low work function of $3.6 \mathrm{eV}$ and a quantum efficiency (QE) measured with $258 \mathrm{~nm}$ UV light of up to $0.2 \%$ [22], which is the highest reported for metal photocathodes.

The design of the photocathode for the SRF gun II is presented in Fig. 6. The cathode plug on top is a cylinder with $10 \mathrm{~mm}$ diameter and $7-8 \mathrm{~mm}$ length, which extends

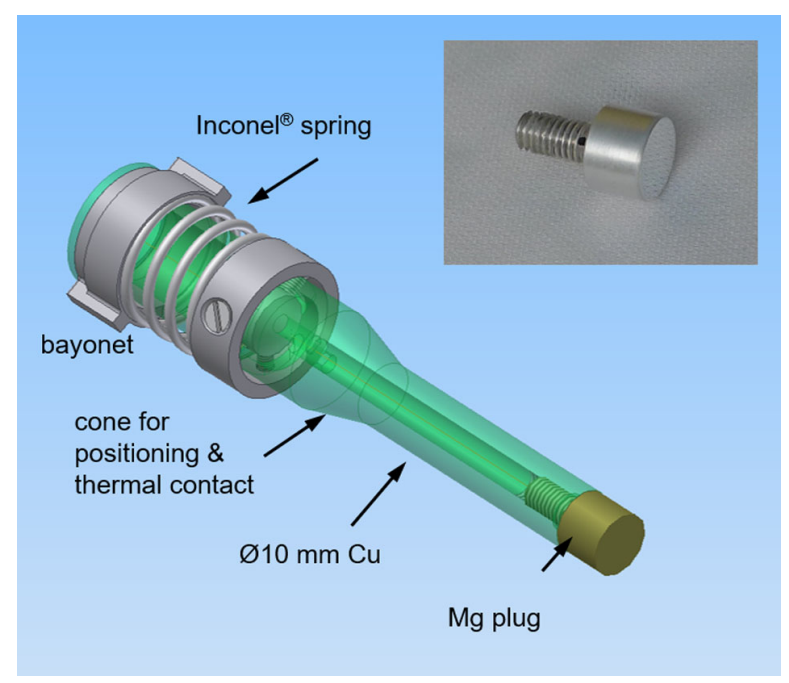

FIG. 6. Geometry of photocathode. The insert shows a Mg plug photograph. into the superconducting cavity. A bayonet fitting holds the cathode in the cooling system as described in Ref. [9]. The cone at the $\mathrm{Cu}$ body of the cathode together with the Inconel ${ }^{\circledR}$ spring realizes a good thermal contact and holds the cathode at $\mathrm{LN}_{2}$ temperature.

The cathode plug consists of pure $99.9 \%$ polycrystalline $\mathrm{Mg}$. The plug is mirrorlike polished, deoxidized, cleaned, and then stored in filtered dry $\mathrm{N}_{2}$ until installed in the cathode transport chamber. After the chemical deoxidation process, the QE of the $\mathrm{Mg}$ cathodes is only about $2 \times 10^{-5}$. In order to reduce the surface work function, the UV drive laser is used for in-vacuum cleaning of the $\mathrm{Mg}$ surface with $100 \mathrm{kHz}$ repetition rate and $100 \mathrm{~mW}$ power. The power density of the laser beam for successful cleaning has to be around $2 \mathrm{~W} / \mathrm{mm}^{2}$. A specific optics setup has been developed $[23,24]$ to perform the cleaning in the cathode transport chamber of the cathode load-lock system. For that reason, the transport chamber is equipped with fused silica windows and a high-voltage feedthrough for an anode collecting the photoemission electrons for QE measurement. The cathodes have to be cleaned in the transport chamber in order to prevent any contamination of the gun cavity.

The laser cleaning leads to a $\mathrm{QE}$ improvement of 2 orders of magnitude, reaching $0.2 \%-0.4 \%$. The cleaning process is very well repeatable [25], so some of the $\mathrm{Mg}$ photocathodes could be cleaned three times. Then, the old $\mathrm{Mg}$ plugs were replaced by new ones. The procedure is to exchange the transport chamber at the gun about one time per year during a shutdown time of ELBE. Beforehand, the new chamber has been filled with three to five photocathodes with new Mg plugs, polished and chemically cleaned, and inspected with respect to particle pollution or scratches in the clean room. After chamber exchange, the load-lock system at the gun is evacuated and vacuum backed. Finally, two Mg cathodes are laser cleaned, QEs are measured, and the best cathode is loaded into the gun. The QE stays stable for a very long time not only in the transport chamber with a vacuum of $\leq 10^{-10}$ mbar but also during SRF gun operation itself. The cathode has always a shiny silver color, but the surface is changed as shown in Fig. 7. The virgin part is the polished mirrorlike surface, while the cleaned part shows a periodic structure created by the scanning pattern of the laser beam.

Up to now, the study of $\mathrm{Mg}$ photocathodes has concentrated on issues affecting a stable and reliable gun operation for terahertz users, i.e., high $\mathrm{QE}$ and long lifetime. Nevertheless, a detailed study of the thermal emittance of $\mathrm{Mg}$ is still in progress. Surface photoemission plays an important role for $\mathrm{Mg}$, and, thus, the thermal emittance is expected to be a factor of 2 lower $(0.5 \mu \mathrm{m} / \mathrm{mm}$ [22]) than predicted by the three-step model for metals assuming volume photoemission [26]. On the other hand, the surface roughness of $100 \mathrm{~nm}$ with a period of 3-20 $\mu \mathrm{m}$ 

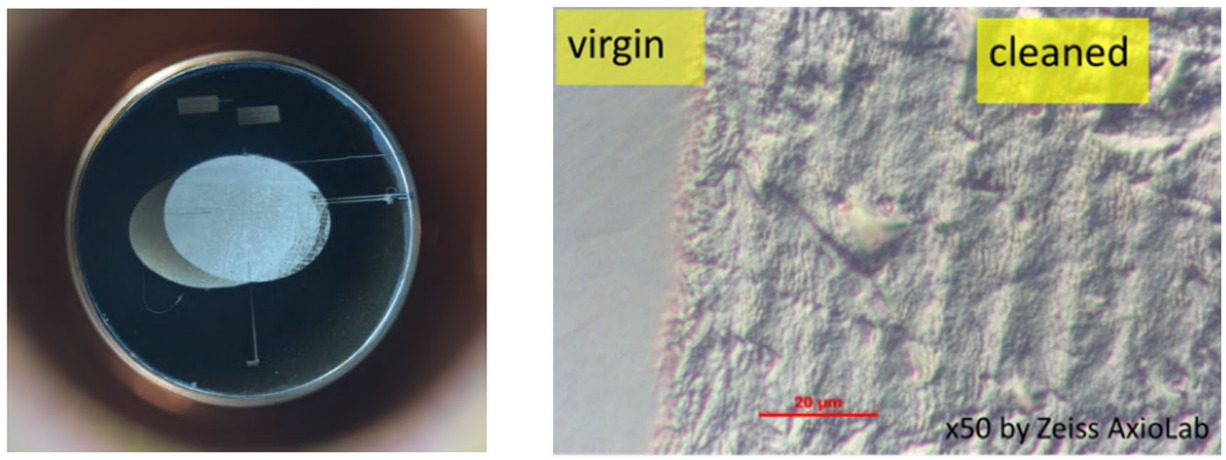

FIG. 7. Photograph of Mg photocathode Mg\#214 after second laser cleaning (left) and surface structure changes after cleaning (optical microscope image, right).

produced by the laser increases the thermal emittance. Applying, e.g., the two-dimensional model by Qian [27] on these values, a growth by $\Delta \varepsilon_{\text {th }}=0.67-0.26 \mu \mathrm{m}$ for $14 \mathrm{MV} / \mathrm{m}$ cathode field and $1 \mathrm{~mm}$ rms laser spot size is expected.

\section{Drive laser system}

The drive laser system for the SRF gun II has been developed by Max Born Institute Berlin. The laser has two channels intended for different pulse repetition rates: One channel is for $13 \mathrm{MHz}$ repetition rate, another for the range $10-500 \mathrm{kHz}$. Both channels produce Gaussian-like pulses in the time domain of 3 and 5 ps FWHM length at $13 \mathrm{MHz}$ and in the kilohertz region, respectively. Average output power is up to $0.5 \mathrm{~W}$ at $262 \mathrm{~nm}$.

The schematic layout of the drive laser system is shown in Fig. 8. The common part of the laser consists of a Nd: glass mode-locked oscillator $(52 \mathrm{MHz})$, followed by a pulse picker that picks every fourth pulse, thus reducing the repetition rate to $13 \mathrm{MHz}$. Then the beam goes into either a fiber amplifier or a regenerative amplifier in case of operating at $13 \mathrm{MHz}$ or at $10-500 \mathrm{kHz}$, respectively.
The final amplification is provided by a multipass amplifier which is common for both channels. At the end, the system splits again into two frequency conversion modules. Each module consists of a lithium triborate (LBO) and a beta barium borate $(\mathrm{BBO})$ crystal that consequently provide IR-to-green and green-to-UV conversion. Because of a big difference in pulse energy at $13 \mathrm{MHz}$ and at $10-500 \mathrm{kHz}$, different focusing is required to achieve the right peak power density within the crystals.

In practice, it turned out that the IR-to-UV conversion is likely to be the main source of instabilities in the output laser beam. When the IR part is rather stable, there are still a few degrees of freedom to play with to optimize the conversion. If not optimized, the crystals can be too much affected by thermal load from the laser beam and, thereby, degrade quickly and/or be too sensitive to small variations in the IR input and in the environment. A significant improvement in both long-term power stability and spatial beam profile (in the kilohertz module) has been achieved rather recently by modifying the beam configuration and placement of the crystals. Figure 9 illustrates the progress in the spatial beam profile.

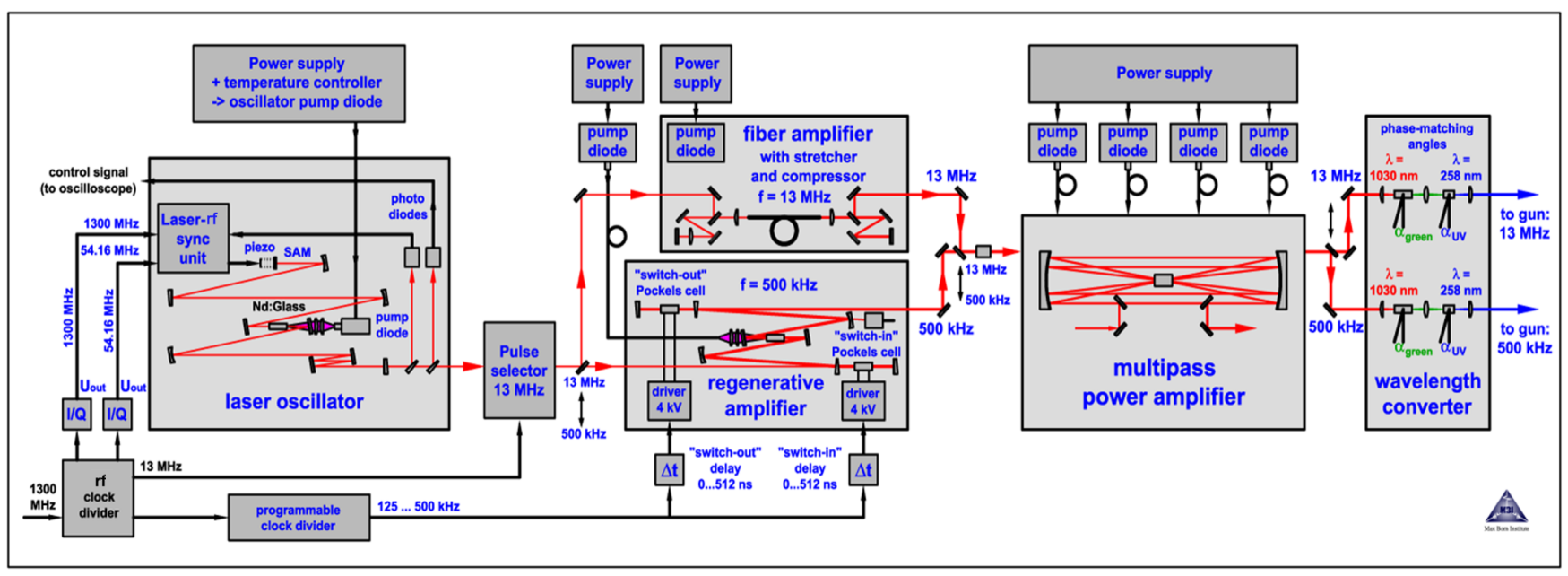

FIG. 8. Scheme of the two-channel laser developed by MBI, Berlin. 

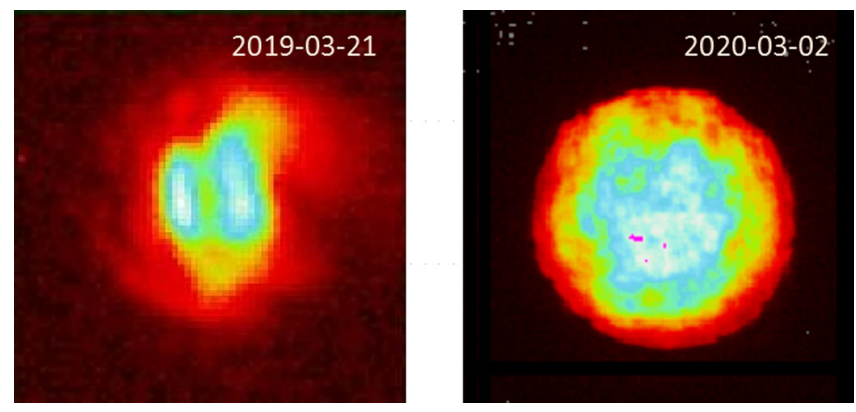

FIG. 9. Laser spot profile on the cathode before and after optimization of the UV conversion (the spot is $3.8 \mathrm{~mm}$ in diameter and is formed as an optical image of a physical aperture on the optical table).

After the laser, an attenuator consisting of a quarter-wave plate and a polarizer is the first optical device on the UV beam path. The attenuator is used to set the required laser power on the photocathode and to compensate for occasional power variations of the laser output. Then, a fast optical mechanical shutter allows one to operate in so-called "macropulse mode" (used when guiding and optimizing the electron beam) where macropulses are $0.2-40 \mathrm{~ms}$ in length and their repetition rate is $1-10 \mathrm{~Hz}$. For the accelerator safety, the beam must be shut down within $2 \mathrm{~ms}$ after a dangerous level of electron beam loss has been detected. Owing to about $0.1 \mathrm{~ms}$ switching time, the optical mechanical shutter allows one to satisfy this condition.

Various photodiodes and power meters and an autocorrelator from APE $\mathrm{GmbH}$ Berlin (recently updated to perform autocorrelation directly in UV as well) are used for diagnostics and constant monitoring of the laser beam. The most recent autocorrelation measurement at UV confirmed the pulse length of $2.3 \mathrm{ps}$ rms (5.3 ps FWHM).

The laser beam transport from the laser table to the photocathode has a length of about $13.5 \mathrm{~m}$. A physical aperture placed at the end of the optical table is imaged onto the cathode with a magnification of 2.5 by two lenses. Attention should be paid to using lenses and other transmitting elements in a pulsed UV laser beam. We have encountered an effect when UV-grade fused silica experiences UV-induced compaction [28], becomes birefringent, and, thus, progressively affects the beam profile. There are different grades of UV fused silica on the market, and we have observed that their resilience to UV laser radiation may differ significantly. At present, we simply avoid using standard UV fused silica elements close to the laser output. The first lens is placed $2 \mathrm{~m}$ after the beam-shaping aperture where the beam is attenuated and rather wide due to some geometrical divergence which it has at the output of the UV conversion and, thus, also after the aperture. Under these conditions, we have not observed any degradation of lenses that perform imaging of the aperture on the cathode. There is a row of apertures of different diameters placed on a motorized linear stage so that one can change between them remotely. A motorized mirror allows one to finely position the laser spot on the cathode. About $2 \mathrm{~m}$ before the cathode, an uncoated wedge picks up about $5 \%$ of laser power for the so-called virtual cathode. The virtual cathode is a Cedoped yttrium aluminum garnet (YAG:Ce) screen placed at the same distance from the wedge as the real cathode so that it allows one to see how the spot on the real cathode looks and how it moves over the cathode with 1:1 ratio. The YAG:Ce screen is imaged on a CCD camera and fluoresces at green when being irradiated by the UV light.

\section{E. Diagnostic beam line and connection to ELBE linac}

A diagnostics beam line is directly connected to the SRF gun [29] (see Fig. 10), whereby its first part also serves for the beam transport into the ELBE accelerator. After the laser input port, a first screen station with a retractable Faraday cup is situated. Following the beam direction, three quadrupoles and a $45^{\circ}$ bending magnet for the ELBE connection are installed. In a straight direction, the second screen station additionally contains a moving slit system for transverse phase space and emittance measurements. After a third screen station, a $180^{\circ}$ spectrometer magnet is placed

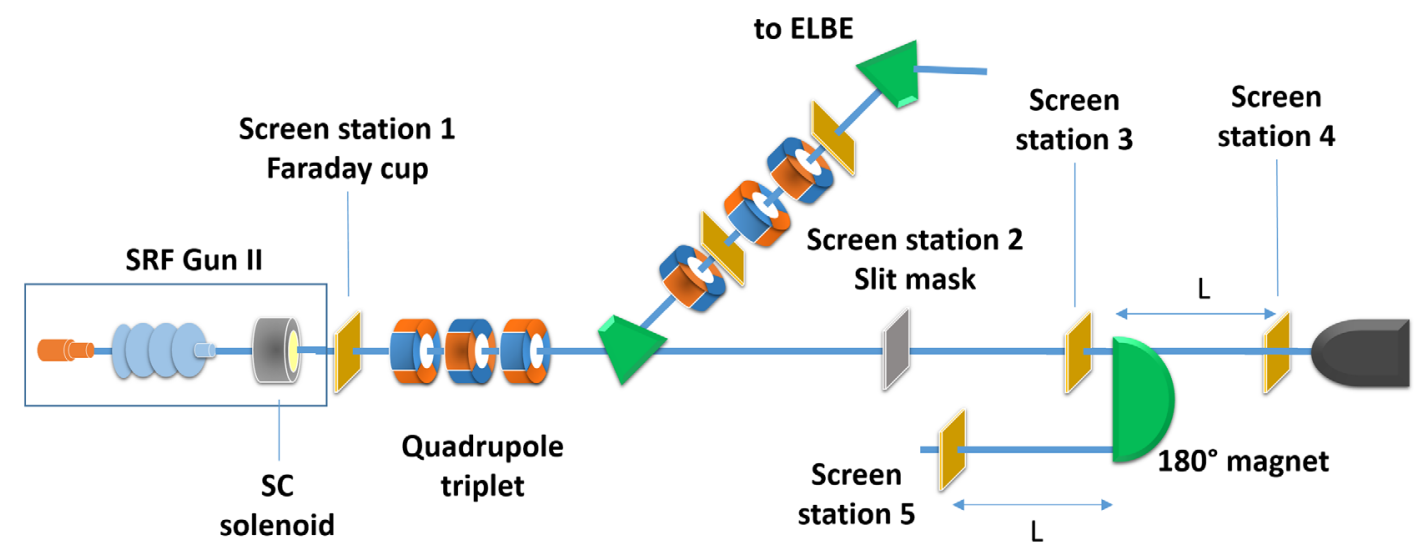

FIG. 10. The diagnostic beamline of the ELBE SRF gun II. 
followed by two screen stations in the straight and $180^{\circ}$ directions, respectively. All screen stations are equipped with YAG:Ce screens. Properly distributed beam position monitors, steering coils, and charge monitors as well as a high-power beam dump at the straight end complete the beam line.

In order to guide the beam to the ELBE main accelerator, an achromatic dogleg structure was built consisting of the dipole in the diagnostic beam line, a second dipole magnet, and a second quadrupole triplet between these dipoles. Thereby, the first quadrupole triplet serves to match the beam to the dogleg, and the second triplet in the dogleg ensures achromatic beam transport.

\section{ELECTRON GUN BEAM PARAMETERS}

The purpose of SRF gun II is to generate a cw beam with higher energy and higher bunch charge but with shorter bunch length and lower transverse emittance than the routinely used dc gun with thermionic cathode at ELBE. Table III summarizes the main beam parameters of SRF gun II. Since 2015, the gun is able to deliver a $4-\mathrm{MeV}$ electron beam with a bunch charge up to $300 \mathrm{pC}$ into the diagnostic beam line and up to $250 \mathrm{pC}$ into ELBE.

Measurements and gun parameter optimization were accompanied by detailed beam dynamical simulations [30]. The charge emission of the photocathode and the beam dynamics in the SRF gun were simulated with ASTRA [31], and further beam transport was computed using ELEGANT [32], in which coherent synchrotron radiation in the bending magnets and the longitudinal space charge effects are considered. These codes were also utilized to develop and optimize the beam transport into the ELBE accelerator for the terahertz radiation production [33] as discussed later in Sec. IV.

The main setting parameters of the gun are the drive laser parameters, which are the pulse length of the temporal Gaussian shaped laser, the laser spot size and its distribution at the cathode, the laser pulse energy, and the laser phase (bunch launches with respect to rf phase). Further parameters are the rf acceleration gradient, the cathode dc

TABLE III. Main beam parameters of SRF gun II with $\mathrm{Mg}$ cathode (gun gradient $8 \mathrm{MV} / \mathrm{m}$ ).

\begin{tabular}{lcc}
\hline \hline Parameter & Simulated [30] & Measured \\
\hline Kinetic energy $(\mathrm{MeV})$ & 4 & 4 \\
Bunch charge (pC) & 200 & $0-300$ \\
Transverse emittance, & $5.8^{\mathrm{a}}$ & $2-15$ \\
$\quad$ rms (mm mrad) & & \\
Energy spread, rms $(\mathrm{keV})$ & $9.8^{\mathrm{a}}$ & $5-25$ \\
Micropulse rate $(\mathrm{kHz})$ & $\ldots$ & $10-500$ \\
Beam current in cw $(\mu \mathrm{A})$ & $\ldots$ & $\leq 30$ \\
Dark current $(\mathrm{nA})$ & $\ldots$ & 40 \\
\hline \hline
\end{tabular}

\footnotetext{
${ }^{\mathrm{a}}$ At $200 \mathrm{pC}$ bunch charge, $65^{\circ}$ laser phase, and $4 \mathrm{~mm}$ diameter laser spot.
}

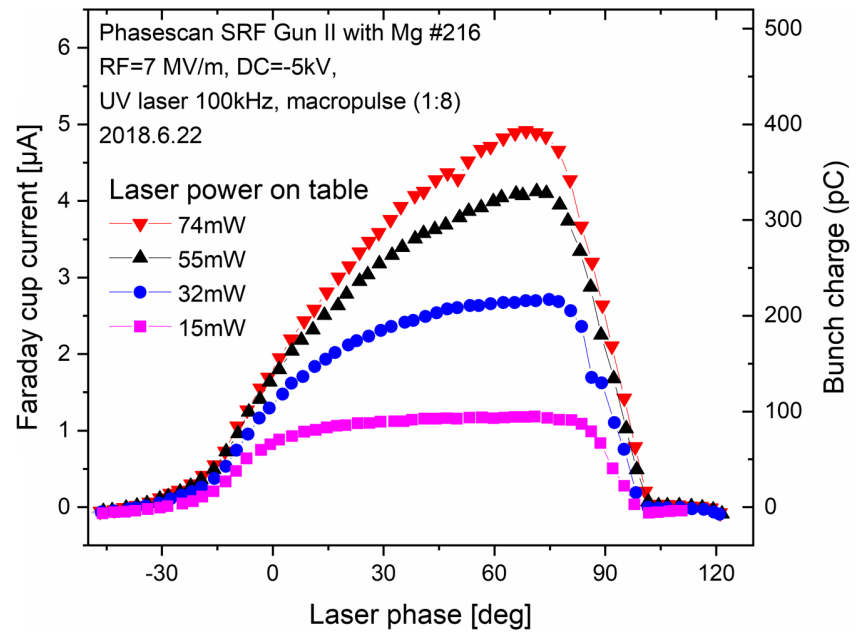

FIG. 11. Measured Faraday cup current and corresponding bunch charge versus drive laser phase. Curves are shown for various UV laser powers.

bias, the cathode retraction position, and the solenoid current.

One of the most significant parameters is the laser phase. Its calibration needs a regular check and correction based on a laser phase scan at low bunch charge (fixed laser power) as presented in Fig. 11. Defining the rf field as sinuslike in time, the beam onset is at $0^{\circ}$ with a strong increase. With growing phase and corresponding extraction field, the Schottky effect reduces the effective work function and, thus, QE and beam current increase. The abrupt drop at about $105^{\circ}$ is due to phase mismatch of the electrons when they reach the TESLA cells of the cavity. The additional field of the negative cathode bias shifts the beam onset in the curve to the left. For higher bunch charges, the photoemission in the SRF gun is dominated by the Schottky effect and space charge. In order to demonstrate the space charge effect, curves are measured with stepwise increased laser power. Thereby, this effect dominates more and more, especially for lower laser phases where the launch field is small. Charges can be extracted up to about $300 \mathrm{pC}$.

Energy and energy spread of the beam were measured with the $180^{\circ}$ dipole magnet in the diagnostics beam line (see Fig. 10). The arrangement of screen 4 in the forward direction downstream from the dipole and screen 5 in the $180^{\circ}$ direction allows a beam spot size correction of the dispersive beam profile measured on screen 5 and, thus, a more accurate energy spread measurement. The kinetic energy for various acceleration gradients is presented in Fig. 12. Measurements were performed up to $9 \mathrm{MV} / \mathrm{m}$. At the standard operation gradient for beam production to ELBE of $8 \mathrm{MV} / \mathrm{m}$, the kinetic energy is about $4 \mathrm{MeV}$. This maximum of beam energy is at a launch phase of about $55^{\circ}$, and the corresponding acceleration field strength at the cathode is then $11.9 \mathrm{MV} / \mathrm{m}$. For terahertz radiation 


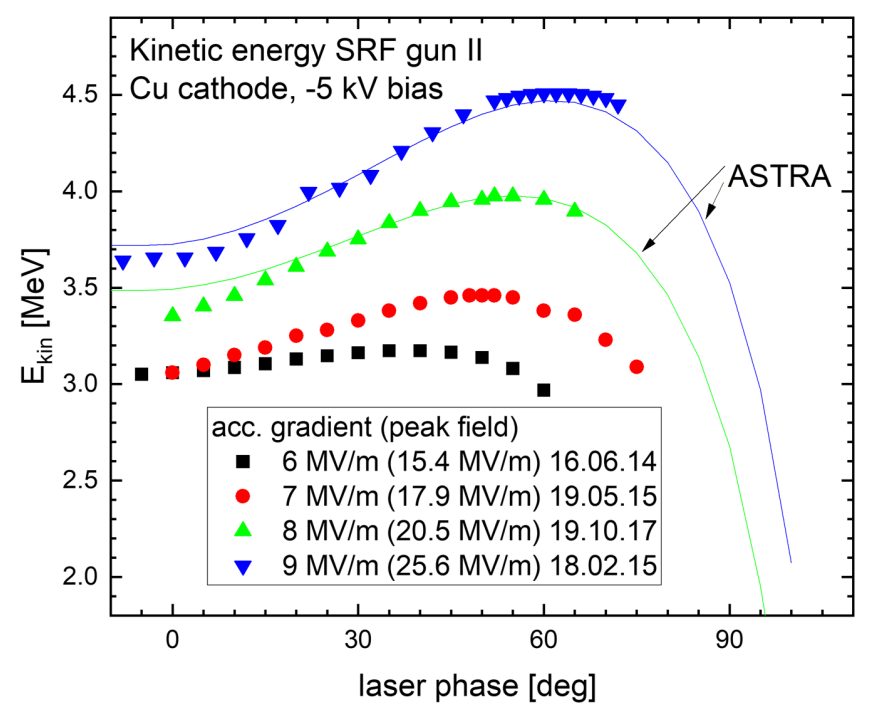

FIG. 12. Kinetic energy at gun exit versus laser phase for acceleration gradients between 6 and $9 \mathrm{MV} / \mathrm{m}$.

production, the gun is operated at lower launch phase of about $45^{\circ}$ and with accordingly $10.3 \mathrm{MV} / \mathrm{m}$ launch field.

The phase of the maximum energy for different gradients increases with the gradient and results from the half-cell design length, i.e., the traveling time in it and the corresponding arrival phase at the TESLA cells. This feature also determines the energy spread behavior as presented in Fig. 13. At laser phases lower than the value for maximum energy, the electron bunches have a negative energy correlation (head has lower energy), and at higher laser phases the energy correlation is positive. Near the point of maximum energy, the rms energy spread has its minimum, but it is increasing with the bunch charge.

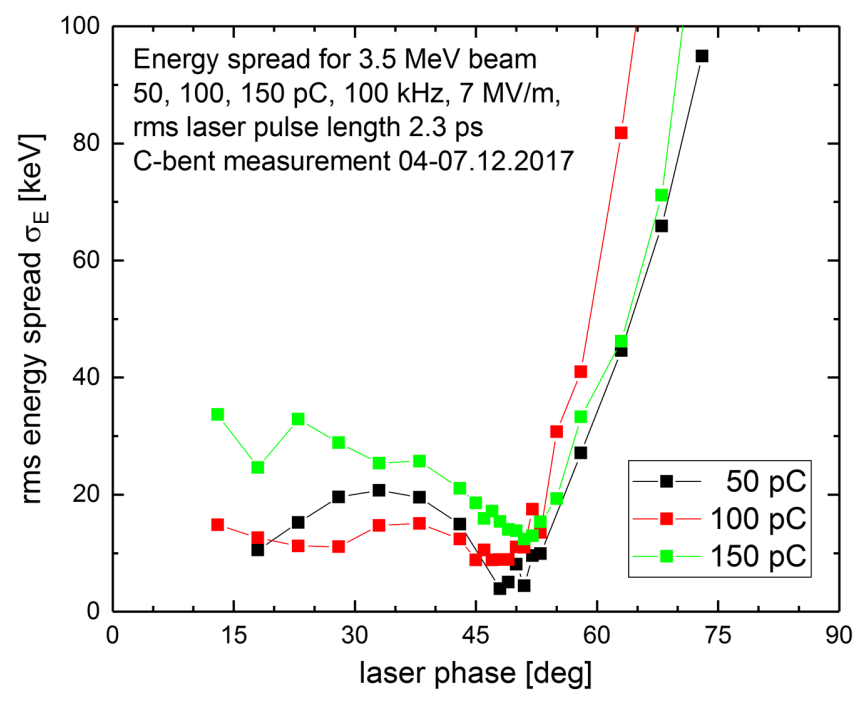

FIG. 13. Energy spread as a function of the laser phase for different bunch charges measured with the $180^{\circ}$ bending magnet of the diagnostics beam line.

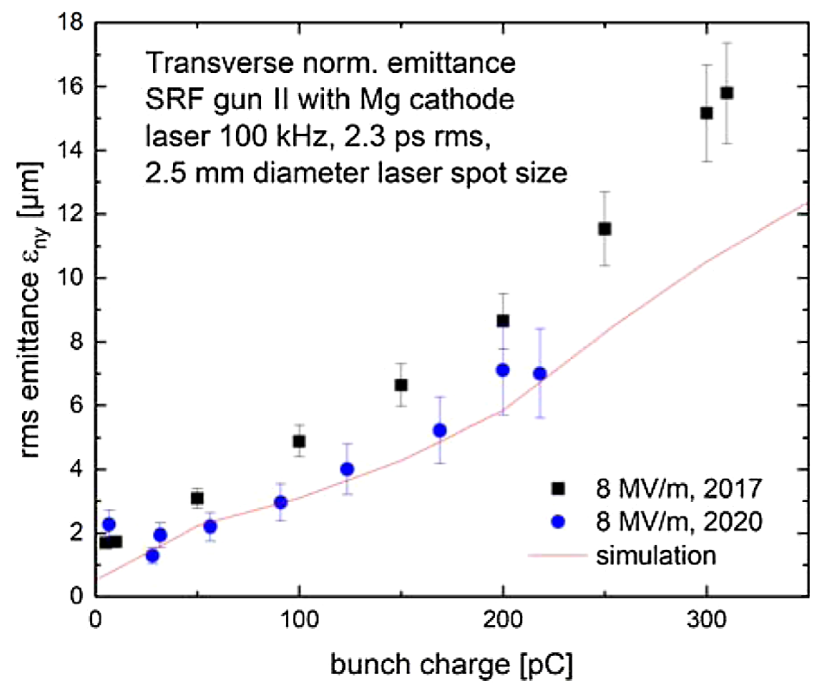

FIG. 14. Measured normalized transverse emittance with slit scan versus bunch charge and comparison with ASTRA simulation results.

The transverse emittance measurements were carried out using a quadrupole scan and slit-mask scan technique. A vertically moving slit is installed in screen station 2 , and the images can be measured with screen station 3 (see Fig. 10). Results of a series of slit-scan measurements for different bunch charges are presented in Fig. 14 for a laser spot of $2.5 \mathrm{~mm}$ diameter and with a Gaussian-shaped laser pulse of $2.3 \mathrm{ps}$ rms. In general, there are three contributions to the transverse emittance: thermal emittance of the photocathode, field effects of the cavity rf field and the solenoid field, and space charge effects. These effects are also included in the ASTRA simulation presented for comparison. The gun and the corresponding simulation parameters, like laser pulse length, laser spot size, and laser phase, are chosen in order to obtain a minimum bunch length of the produced bunch for $200 \mathrm{pC}$, which is the most important criteria for terahertz production application, described in Sec. IV. For that reason, the transverse emittance values are relatively high in simulation and in measurement. The differences between experimental and simulation values can be caused by a number of effects. First, as discussed above, the higher thermal emittance due to the cathode roughness is not included in the simulation and could explain the higher emittance at low bunch charges. Second, the simulation was performed for a perfect flattop laser profile, but the experimental transversal laser profile at the cathode was very inhomogeneous and asymmetric in the measurement in year 2017 (see Fig. 9). A similar effect could also come from the QE inhomogeneity of the photocathodes. But, as visible in the 2020 results, the improvement of the laser profile results in a good agreement with the simulation results. Finally, we observed astigmatism, which may be caused by the asymmetry of the rf field due to the coupler and imperfection or misalignment 


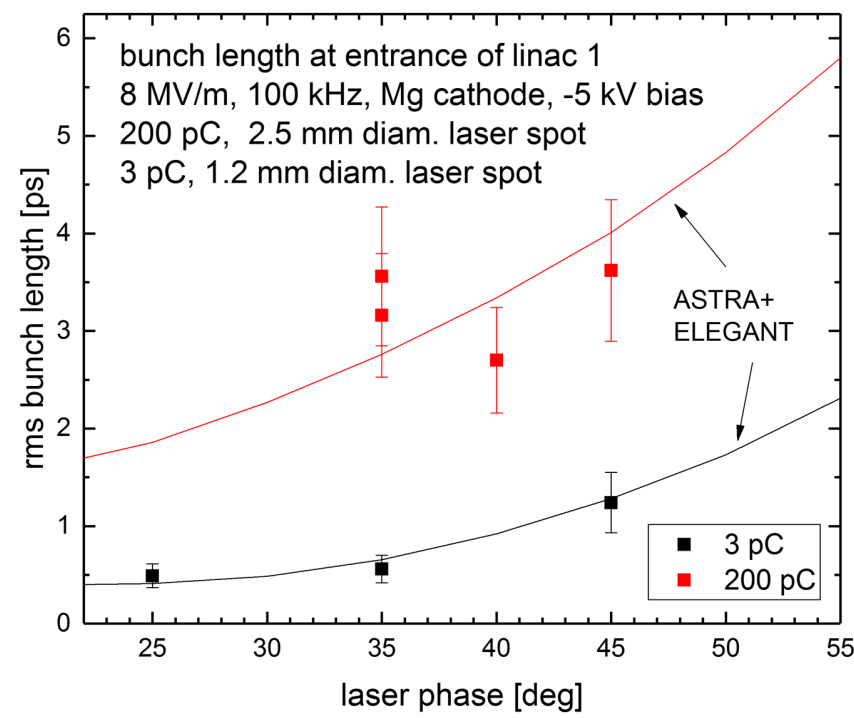

FIG. 15. Bunch length versus laser phase for 3 and $200 \mathrm{pC}$ at the entrance of accelerator module 1 (cavity $\mathrm{C} 1$ ) measured with the cavity phase scan method.

of the solenoid. These effects have been partly compensated now with quadrupole correctors similar to the design proposed in Ref. [34] and used at the photo injector test facility (PITZ) photoinjector.

At present, there is no bunch length measurement method available in the diagnostic beam line. But, by guiding the beam via the dogleg to the first linac module of ELBE, a phase variation of the first cavity and a following dipole can be utilized for a longitudinal phase space measurement. This phase scan method is described in Refs. [35,36] and is quasi the longitudinal analogon of the quadrupole scan. Since it samples the beam at the first cavity, usually a correction is needed to obtain the values at the position of electron gun exit. However, here we are directly interested in the measurement values at entrance of linac module 1, because the later bunch compression requires bunches as short as possible at this position. Figure 15 presents the measurement results for bunches of 3 and $200 \mathrm{pC}$. It can be seen that the negative longitudinal correlation that the bunch has at small laser phases leads to bunch compression (velocity bunching) for low charges and preserves the initial bunch length for $200 \mathrm{pC}$ bunches (laser with 2.3 ps rms Gaussian profile).

\section{SRF GUN APPLICATION FOR TERAHERTZ RADIATION PRODUCTION}

\section{A. TELBE facility at HZDR}

Terahertz (THz) radiation, which is located between the infrared light and microwaves in the frequency domain, attracts much interest due to its wide applications in biomedicine, imaging and security, communication, and condensed matter physics. Intense $\mathrm{THz}$ sources are new tools to study nonlinear $\mathrm{THz}$ field-induced carrier dynamics in condensed matter like photoexcitation, intense THzpump and THz-probe experiments, THz-induced electron field emission, switching of superconductors, magnetic and polarization switching, or nonlinear response of metamaterials [37,38]. In accelerator-based radiation sources, the use of relativistic electrons and the use of superradiance, i.e., ultrashort electron bunches (tenths of picoseconds) in which electrons radiate in phase, have been the key elements. The TELBE facility at the ELBE radiation source was established in 2013 [7,39] and started user operation in 2016. TELBE combines superradiant $\mathrm{THz}$ radiation sources with a superconducting accelerator operating in cw mode and with pulse repetition rates up to several megahertz. Therefore, a high brilliant $\mathrm{THz}$ source with high average power, high pulse energy, and high flexibility in wavelength tuning and in pulse time structure can be offered to users. TELBE has two radiation stages, which can be simultaneously used. One generates broadband, single-cycle coherent diffraction radiation (CDR), and the second one tunable small bandwidth coherent undulator radiation (CUR). Both kinds of radiation are transported to a $\mathrm{THz}$ user lab, where the radiation can be characterized and applied for a variety of experimental setups. The radiation is created by relativistic, subpicosecond electron bunches. For coherent radiation, that means the longitudinal bunch length is significantly shorter than the wavelength, and the intensity grows with the square of the number of electrons. Therefore, the spectral angular distribution of the radiation of an electron bunch can be written as

$$
\frac{d^{2} I}{d \omega d \Omega}=N\{1+(N-1) F(\omega)\} \frac{d^{2} I_{s e}}{d \omega d \Omega} .
$$

Here $N$ denotes the number of electrons in the bunch, $\omega$ is the radiation angular frequency, and $d^{2} I_{s e} / d \omega d \Omega$ is the spectral angular intensity for a single electron. The bunch longitudinal form factor $F(\omega)$ of the normalized charge distribution $\rho(z)$ is given by

$$
F(\omega)=\left|\int \rho(z) e^{-i \omega z / c} d z\right|^{2}
$$

and has a value between zero and one. As can be seen in Eq. (1), the spectral power splits into an incoherent and a coherent part. For the coherent part, the quadratic increase with bunch charge sustains as long as the form factor does not decrease. Consequently, a higher bunch charge needs successful bunch compression.

\section{B. Beamline and beam transport}

The gun acceleration gradient was chosen to be $8.0 \mathrm{MV} / \mathrm{m}$, which is the maximum value to hold field emission, helium consumption, and dark current to acceptable levels, and it also ensures a stable operation of the gun. 
With this setting, the rf of the gun runs without disruptions for typical user beam times of one week or even longer. The $\mathrm{THz}$ application requires bunch charges as high as possible and short electron bunch lengths already at the gun exit, as will be discussed later. The transverse emittance plays a less important role. Parameters forming a good compromise for $\mathrm{THz}$ production are a $2.3 \mathrm{ps}$ rms length of the Gaussian-shaped laser pulse, a laser spot size of $4 \mathrm{~mm}$ diameter at the cathode, and bunch charges of $200 \mathrm{pC}$ and recently of $250 \mathrm{pC}$. The photocathode was retracted by $1.3 \mathrm{~mm}$ and had a bias of $-5 \mathrm{kV}$. The standard working point of the gun is at a laser phase of about $50^{\circ}$, which is characterized by the maximum of electron energy and the minimum of rms energy spread. The latter is important for the following beam transport through the dispersive dogleg section. However, for $\mathrm{THz}$ production the laser phase is set to a different value of about $45^{\circ}$ in order to obtain the required shorter bunch length at gun exit.

For $\mathrm{THz}$ production at ELBE, the beam line layout with the SRF gun, dogleg, the two accelerator modules, magnetic chicane, undulator, and the path to the beam dump is schematically shown in Fig. 16. The SC solenoid and the first quadrupole triplet downstream from the SRF gun are set to match the beam for the dogleg. The quadrupoles inside the dogleg ensure achromaticity. In the first ELBE linac module (cavities $\mathrm{C} 1$ and $\mathrm{C} 2$ ), the electron bunches are accelerated to $15.5 \mathrm{MeV}$ with on-crest rf phases. The second ELBE linac module (cavities C3 and C4) serves for further acceleration as well as to create the energy chirp by off-crest operation. The final beam energy of about $26 \mathrm{MeV}$ is held constant; i.e., any rf phase variations of $\mathrm{C} 3$ and $\mathrm{C} 4$ are compensated by corresponding changes of the gradients. The energy chirp, i.e., a correlation between particle momentum and longitudinal coordinate in the bunch, is needed for the following magnetic bunch compression in the chicane. The chicane consists of four parallel face dipole magnets. After the chicane, the first $\mathrm{THz}$ radiation source is situated, which produces CDR and consists of a screen with aperture of $4 \mathrm{~mm}$ diameter. The following second $\mathrm{THz}$ source can produce CUR in the spectral range of 0.1-3 THz. The electromagnetic undulator has eight periods with a period length of $300 \mathrm{~mm}$.

\section{Electron beam properties}

The following discussion is focused on the longitudinal properties of the beam, since the final bunch length at the radiation stations determines the coherence and, thus, the output power of $\mathrm{THz}$ radiation. Figure 17 presents simulation results for the evolution of the bunch length $\sigma_{t}$ along the beam line. The curves are for three different laser phases. Beside the laser pulse length itself, the laser phase has a large effect on the bunch length at the entrance of linac 1. This phase determines whether the bunch is

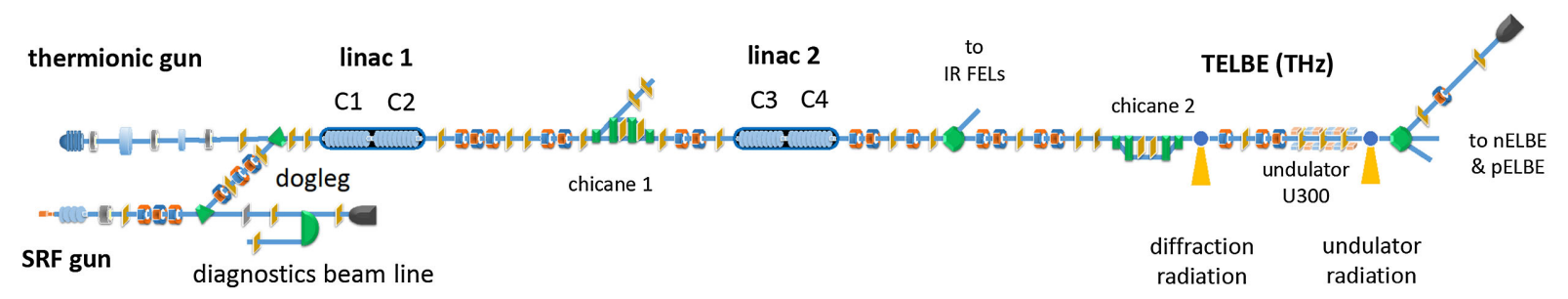

FIG. 16. Beamline layout for THz radiation production at ELBE.

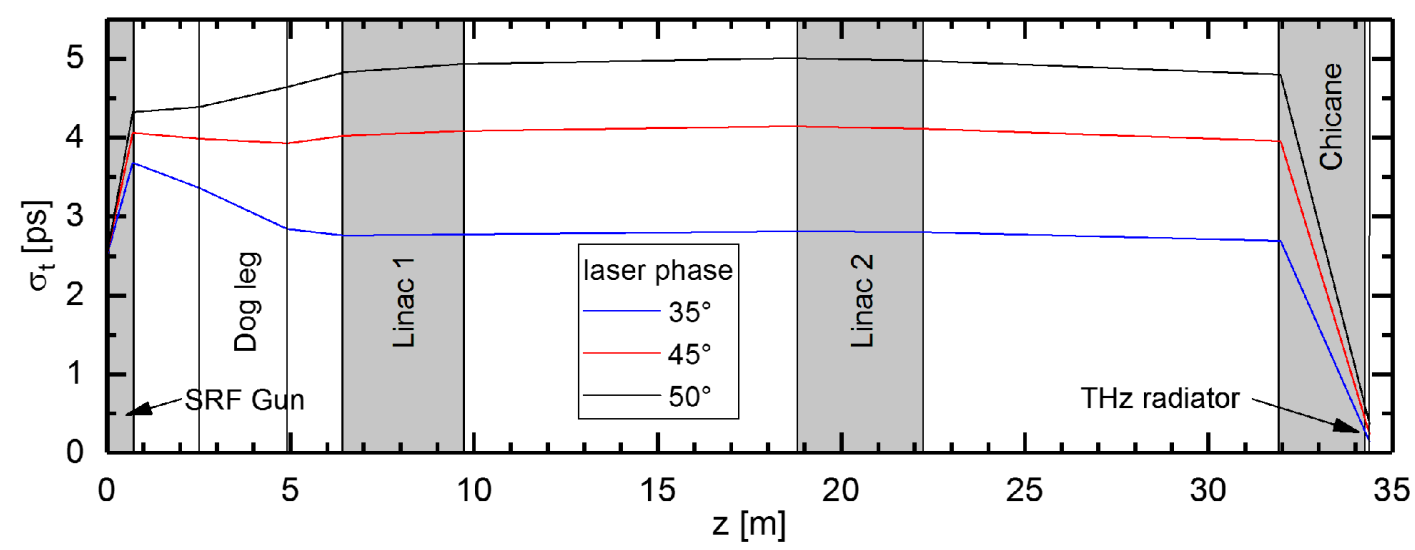

FIG. 17. Evolution of rms bunch length in the beam line from SRF gun to THz radiator for three different SRF gun drive laser phases. The simulation results are obtained from ASTRA and ELEGANT with typical experimental gun parameters: $20.5 \mathrm{MV} / \mathrm{m}$ gun peak field on axis, $4 \mathrm{~mm}$ laser spot diameter, $2.3 \mathrm{ps}$ rms laser pulse length, and $200 \mathrm{pC}$ bunch charge. 


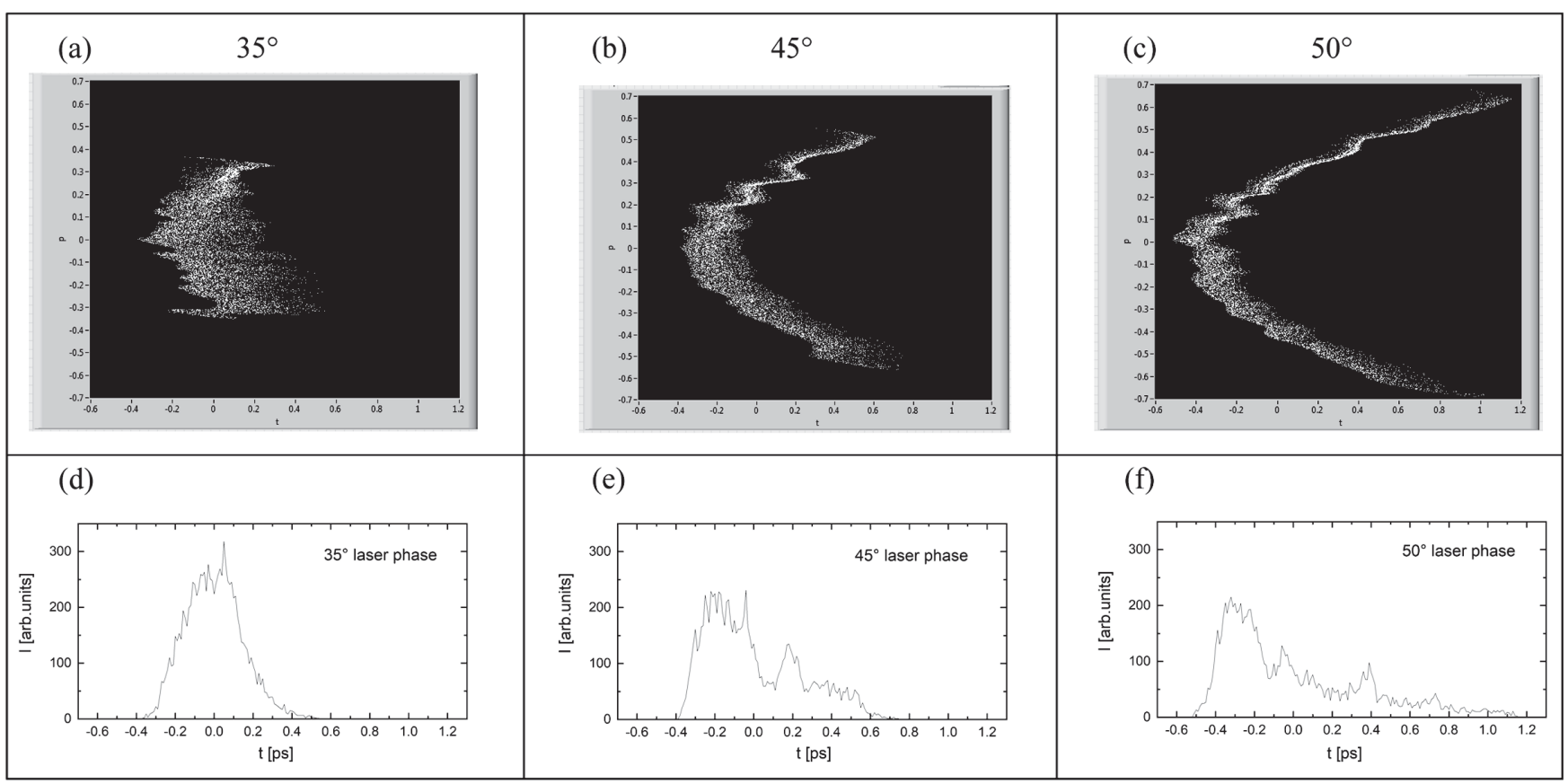

FIG. 18. Simulation results of longitudinal phase space distribution for $35^{\circ}$ laser phase (a), $45^{\circ}$ laser phase (b), and $50^{\circ}$ laser phase (c) at the $\mathrm{THz}$ radiator position. The associated longitudinal charge distributions are shown in the plots (d) to (f).

compressed (velocity bunching) or not within the gun and in the dogleg section, which means the bunch lengths are $2.8 \mathrm{ps}$ for $35^{\circ}$ and $4.8 \mathrm{ps}$ for $50^{\circ}$ laser phase. As a consequence of shorter bunch lengths, there are decreasing nonlinear energy spread contributions from linac 1 and smaller correlated energy spread values at optimum chirp phase after linac 2. In Fig. 18, the simulation results of the longitudinal phase space after compression at the $\mathrm{THz}$ radiator position are presented. The nonlinear energy spread produced by linac 1 gives the $\mathrm{C}$-shape distributions in the cases of the $50^{\circ}$ and $45^{\circ}$ laser phases. These shapes

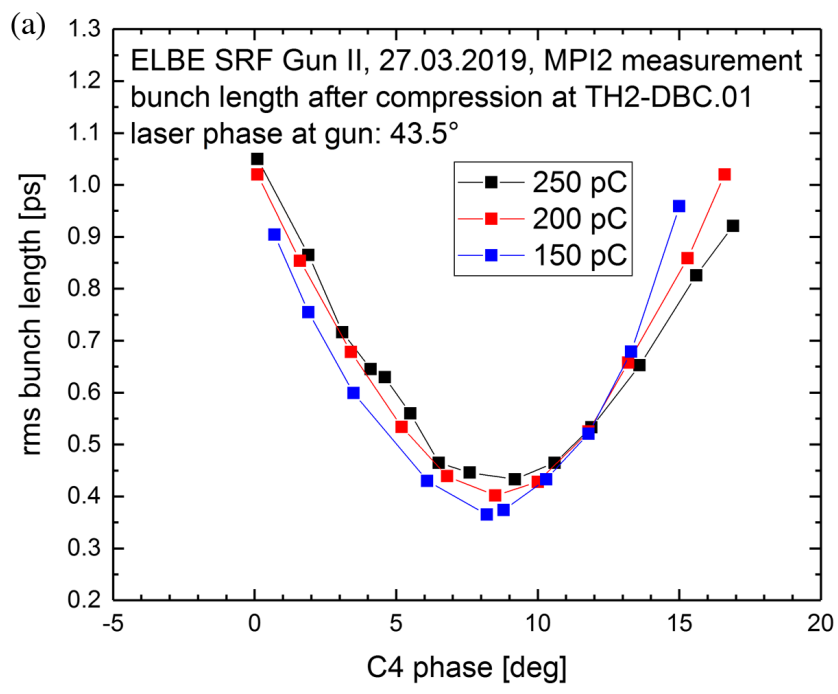

define the final bunch length. Only for the $35^{\circ}$ case is the bunch short enough at linac 1 that this shape is not visible, and the remaining uncorrelated energy spread determines the final bunch length.

Experimentally, laser phases between $40^{\circ}$ to $45^{\circ}$ were applied in most of the cases, for which sufficiently compressed electron pulses could be produced. The compressed electron bunch length was measured with a Martin-Puplett interferometer [40] analyzing coherent transition radiation in front of the undulator. Bunch length results as a function of compression (C4 phase) for different bunch charges and

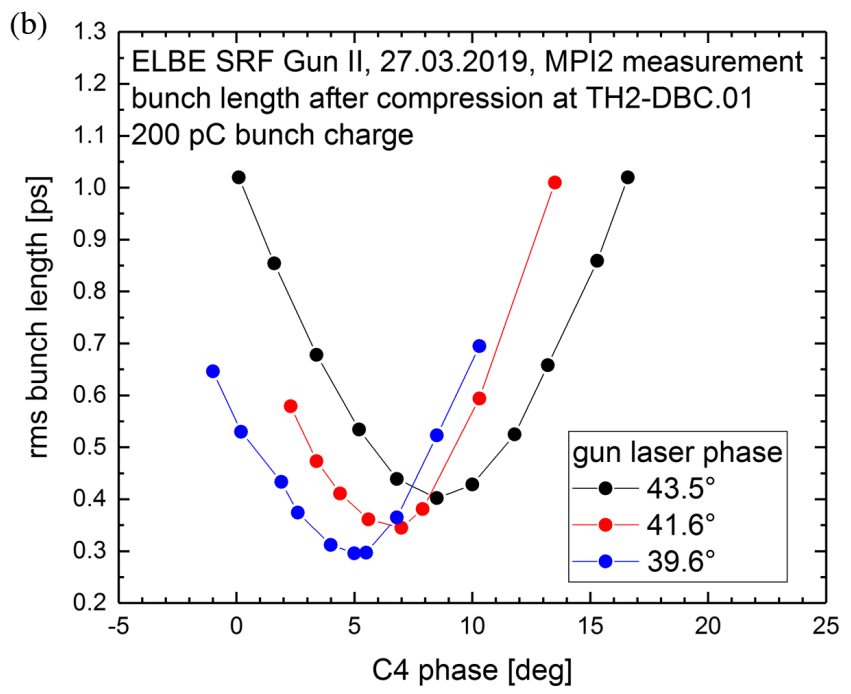

FIG. 19. Bunch length measurement after bunch compression with magnetic chicane versus cavity C4 phase for different bunch charges (a) and different drive laser phases (b). 


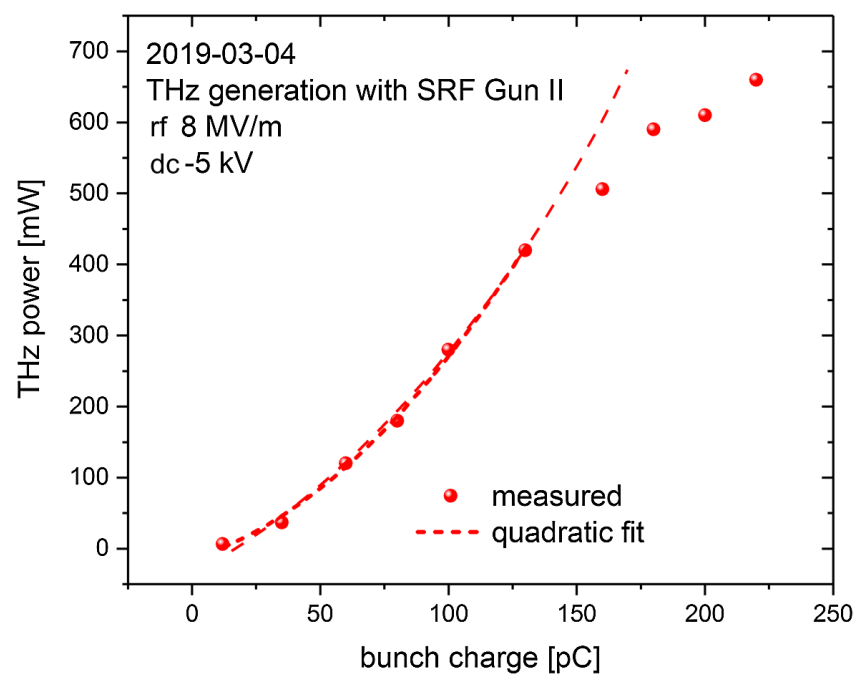

FIG. 20. Dependence of undulator radiation power at $0.7 \mathrm{THz}$ on electron bunch charge.

gun drive laser phases are presented in Fig. 19. In agreement with the simulations, these measurements show that a lower laser phase results in shorter compressed bunches.

\section{Results of $\mathrm{THz}$ radiation production}

The measured $\mathrm{THz}$ power as a function of bunch charge is shown in Fig. 20. The bunch charge is varied by changing the photocathode laser pulse energy. Up to $150 \mathrm{pC}$, a clear quadratic dependency is visible, whereas for higher charges the behavior is more linear. The reason is an increase in bunch length, but it also needs a more careful optimization of beam transport and compression at each charge value due to increasing space charge effects, which was not done in this measurement. For compression, the chicane was set on a fixed value in order to sustain the transverse beam

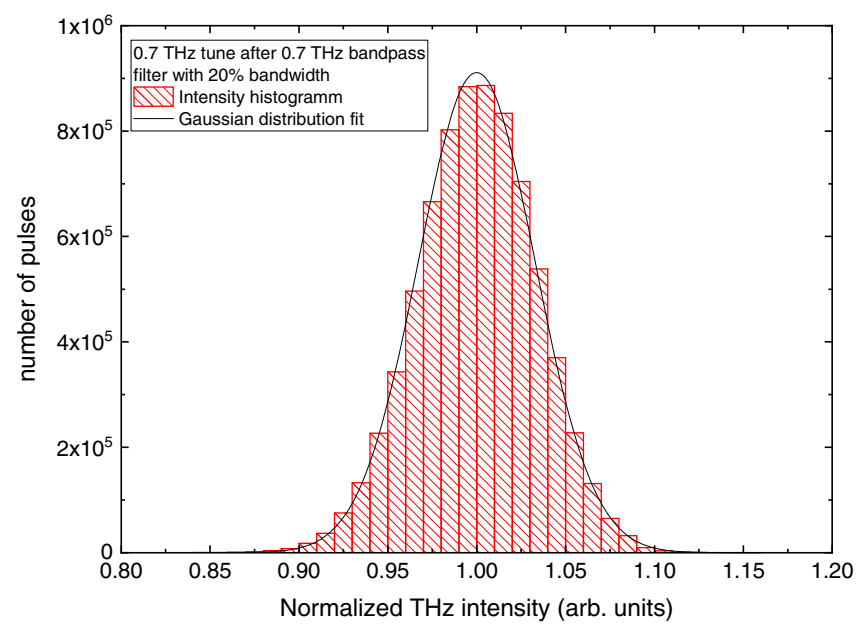

FIG. 21. Normalized $\mathrm{THz}$ intensity histogram for a typical 10 min measurement. The standard deviation of the Gaussian distribution is 0.0328 .

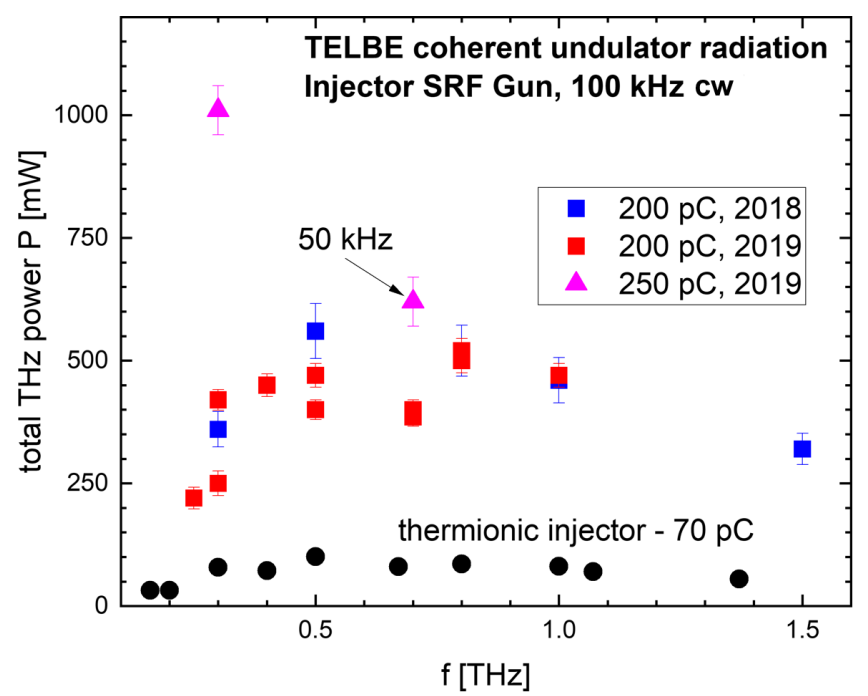

FIG. 22. Undulator radiation power produced in cw operation for different frequencies at the TELBE facility.

parameters. Then the phases of $\mathrm{C} 3$ and $\mathrm{C} 4$ were tuned for optimal bunching together with the gradients holding the final beam energy at a constant value. Fine-tuning was done with the phase of cavity $\mathrm{C} 4$. Figure 21 shows the normalized intensity histogram of the $\mathrm{THz}$ intensity over a typical measurement duration of $10 \mathrm{~min}$. This measurement contains data points from $7.5 \times 10^{6} \mathrm{THz}$ pulses (repetition rate of $50 \mathrm{kHz}$ ). The data were obtained using a fast pyroelectric detector, which transforms the incident $\mathrm{THz}$ radiation into a voltage signal that is read out using a fast analog-to-digital converter. Before impinging on the detector, the narrowband $\mathrm{THz}$ radiation from the undulator source propagates through a $\mathrm{THz}$ bandpass filter with a central frequency of $0.7 \mathrm{THz}$ and a bandwidth of $20 \%$ (FWHM). The distribution of $\mathrm{THz}$ intensities is clearly Gaussian with a standard deviation of about 0.03 , demonstrating the high-intensity stability of the $\mathrm{THz}$ source.

Figure 22 presents the $\mathrm{cw} \mathrm{THz}$ power within a frequency range of $0.15-1.5 \mathrm{THz}$ produced during user shifts with 50 and $100 \mathrm{kHz}$ pulse repetition rate in the years 2018 and 2019. Typical power values for $200 \mathrm{pC}$ bunch charge are between 300 and $500 \mathrm{~mW}$. The maximum values are for $250 \mathrm{pC}$ charge with $1 \mathrm{~W}$ at $0.3 \mathrm{THz}(10 \mu \mathrm{J})$ and $620 \mathrm{~mW}$ with $50 \mathrm{kHz}$ at $0.7 \mathrm{THz}(12.4 \mu \mathrm{J})$. Before 2018, the thermionic injector had been used for $\mathrm{THz}$ production at ELBE. In comparison, the $\mathrm{THz}$ output applying the SRF gun could be increased up to a factor of 5 for $200 \mathrm{pC}$ and by a factor of 10 for $250 \mathrm{pC}$ bunch charge.

\section{CONCLUSIONS}

As an $R \& D$ effort to explore a new cw injector for the ELBE accelerator, SRF gun II is actually a successful prototype and an excellent demonstration of SRF gun technology for user operation. With this new injector, 
the ELBE center is able to provide ultrashort electron bunches with significantly higher charge to the $\mathrm{THz}$ facility than delivered by the thermionic de gun.

Until now (April 2020), the SRF gun has been applied for the ELBE accelerator in $119 \mathrm{THz}$ shifts and 18 shifts for other purposes, i.e., for about $1640 \mathrm{~h}$ beam time. During these months, two Mg photocathodes were operated in the SRF gun and delivered a total charge of $50.6 \mathrm{C}$. The advantages of $\mathrm{Mg}$ photocathodes, consisting of solid $\mathrm{Mg}$ plugs, are easy fabrication, a relatively high QE after laser cleaning, and a low risk of surface contamination of the superconducting cavity. And neither a degradation of cavity performance nor an increase of field emission was observed during the operation with $\mathrm{Mg}$ photocathodes since 2016.

\section{ACKNOWLEDGMENTS}

We thank the whole ELBE team for their help with this project. The work was partly supported by the German Federal Ministry of Education and Research (BMBF) Grant No. 05K12CR1 and Deutsche Forschungsgemeinschaft (DFG) project (XI 106/2-1).

[1] P. Michel, ELBE Center for high-power radiation sources, J. Large-Scale Res. Facil. 2, A39 (2016).

[2] C. Hernandez-Garcia, D. Bullard, F. Hannon, Y. Wang, and M. Poelker, High voltage performance of a dc photoemission electron gun with centrifugal barrel-polished electrodes, Rev. Sci. Instrum. 88, 093303 (2017).

[3] M. Yamamoto and N. Nishimori, High voltage threshold for stable operation in a dc electron gun, Appl. Phys. Lett. 109, 014103 (2016).

[4] A. Arnold and J. Teichert, Overview on superconducting photoinjectors, Phys. Rev. Accel. Beams 14, 024801 (2011).

[5] F. Sannibale, D. Filippetto, H. Qian, C. Mitchell, F. Zhou, T. Vecchione, R. K. Li, S. Gierman, and J. Schmerge, High-brightness beam tests of the very high frequency gun at the Advanced Photo-injector EXperiment test facility at the Lawrence Berkeley National Laboratory, Rev. Sci. Instrum. 90, 033304 (2019).

[6] J. Klug et al., Development of a neutron time-of-flight source at the ELBE accelerator, Nucl. Instrum. Methods Phys. Res., Sect. A 577, 641 (2007).

[7] B. Green et al., High-field high-repetition-rate sources for the coherent $\mathrm{THz}$ control of matter, Sci. Rep. 6, 22256 (2016).

[8] A. Jochmann et al., High Resolution Energy-Angle Correlation Measurement of Hard X Rays from LaserThomson Backscattering, Phys. Rev. Lett. 111, 114803 (2013).

[9] A. Arnold et al., Development of a superconducting radio frequency photoelectron injector, Nucl. Instrum. Methods Phys. Res., Sect. A 577, 440 (2007).

[10] J. Teichert et al., Free-electron laser operation with a superconducting radio-frequency photoinjector at ELBE,
Nucl. Instrum. Methods Phys. Res., Sect. A 743, 114 (2014).

[11] X. J. Wang and P. Musumeci, Report of the Basic Energy Sciences Workshop on the Future of Electron Sources, SLAC National Accelerator Laboratory, 2016.

[12] T. Kamps, bERLinPro project team, SRF gun development for energy recovery linac applications, in High-Brightness Sources, and Light-Driven Interactions, OSA technical digest (Optical Society of America, Washington, D.C., 2016), paper ET1A.3. See also M. Abo-Bakr et al., Progress report of the Berlin energy recovery project bERLinPro, in Proceedings of the 2015 International Particle Accelerator Conference, Richmond, VA (JACoW, Geneva, 2015), p. 1438.

[13] E. Vogel et al., SRF gun development at DESY, in Proceedings of the 29th Linear Accelerator Conference LINAC2018, Beijing, China (JACoW, Geneva, 2015), p. 105.

[14] L.W. Feng et al., Ultrafast electron diffraction with megahertz $\mathrm{MeV}$ electron pulses from a superconducting radio-frequency photoinjector, Appl. Phys. Lett. 107, 224101 (2015).

[15] H. Vennekate, A. Arnold, P. Lu, P. Murcek, J. Teichert, and R. Xiang, Emittance compensation schemes for a superconducting rf injector, Phys. Rev. Accel. Beams 21, 093403 (2018).

[16] P. Murcek, A. Arnold, H. Büttig, D. Janssen, M. Justus, P. Michel, G. Staats, J. Teichert, R. Xiang, and P. Kneisel, Modified SRF photoinjector for the ELBE at HZDR, in Proceedings of the 15th International Conference on RF Superconductivity (SRF2011), Chicago, IL (JACoW, Geneva, 2011), p. 39.

[17] A. Arnold, P. Murcek, J. Teichert, R. Xiang, P. Kneisel, L. Turlington, M. Stirbet, and G. Eremeev, Fabrication, tuning, treatment and testing of two 3.5 cell photo-injector cavities for the ELBE linac, in Proceedings of the 15th International Conference on RF Superconductivity (SRF2011), Chicago, IL (JACoW, Geneva, 2011), p. 405.

[18] A. Arnold, M. Freitag, P. Murcek, J. Teichert, H. Vennekate, R. Xiang, P. Kneisel, G. Ciovati, and L. Turlington, RF performance results of the 2nd ELBE srf gun, in Proceedings of the 17th International Conference on Superconductivity (SRF 2015), Whistler, Canada (JACoW, Geneva, 2015), p. 1227.

[19] A. Arnold, M. Freitag, P. Murcek, J. Teichert, H. Vennekate, R. Xiang, P. Kneisel, G. Ciovati, and L. Turlington, Commissioning results of the 2nd 3.5 cell SRF gun for ELBE, in Proceedings of the 27th Linear Accelerator Conference (LINAC2014), Geneva, Switzerland (JACoW, Geneva, 2014), p. 578.

[20] E. T. Tulu, U. van Rienen, and A. Arnold, Different countermeasures of electron amplification in the photocathode unit, in Proceedings of the 5th International Particle Accelerator Conference (IPAC2014), Dresden, Germany (JACoW, Geneva, 2014), p. 652.

[21] R. Xiang, A. Arnold, T. Kamps, P. Lu, P. Michel, P. Murcek, H. Vennekate, G. Staats, and J. Teichert, Experimental studies of dark current in a superconducting rf photoinjector, Phys. Rev. Accel. Beams 17, 043401 (2014). 
[22] H. J. Qian, J. B. Murphy, Y. Shen, C. X. Tang, and X. J. Wang, Surface photoemission in a high-brightness electron beam radio frequency gun, Appl. Phys. Lett. 97, 253504 (2010).

[23] R. Xiang, A. Arnold, P. Michel, P. Murcek, J. Teichert, P. $\mathrm{Lu}, \mathrm{H}$. Vennekate, and P. Patra, Improvement of photoemission efficiency of magnesium photocathodes, in Proceedings of the 2017 International Particle Accelerator Conference, Copenhagen, Denmark (JACoW, Geneva, 2017), p. 500.

[24] R. Xiang, A. Arnold, P. Lu, P. Murcek, J. Teichert, and H. Vennekate, Study of magnisium photocathodes for superconducting rf photoinjectors, in Proceedings of the 2018 International Particle Accelerator Conference, Vancouver, Canada (JACoW, Geneva, 2018), p. 4142.

[25] R. Xiang, A. Arnold, P. Murcek, J. Teichert, and J. Schaber, Metal and semiconductor photocathodes in HZDR SRF gun, in Proceedings of the 63th ICFA Advanced Beam Dynamics Workshop on Energy Recovery Linac (ERL19), Berlin, Germany (JACoW, Geneva, 2019), p. 142.

[26] D. H. Dowell and J. F. Schmerge, Quantum efficiency and thermal emittance of metal photocathodes, Phys. Rev. Accel. Beams 12, 074201 (2009).

[27] H. J. Qian, C. Li, Y. C. Du, L. X. Yan, J. F. Hua, W. H. Huang, and C.X. Tang, Experimental investigation of thermal emittance components of copper photocathode, Phys. Rev. Accel. Beams 15, 040102 (2012).

[28] R. E. Schenker and W. G. Oldham, Ultraviolet-induced densification in fused silica, J. Appl. Phys. 82, 1065 (1997).

[29] T. Kamps et al., Electron beam diagnostics for a superconducting radio frequency photoelectron injector, Rev. Sci. Instrum. 79, 093301 (2008).

[30] P. Lu, Optimization of an SRF gun for high bunch charge applications at ELBE, Ph.D. thesis, TU Dresden, 2017.

[31] K. Flöttmann, ASTRA: A space charge tracking algorithm, manual, version 3, 2011, update April 2014, http:/www .desy.de/ $\sim$ mpyflo/.
[32] M. Borland, ELEGANT: A Flexible SDDS-Compliant Code for Accelerator Simulation, Advanced Photon Source Report No. LS-287, 2000.

[33] P. Lu, A. Arnold, J. Teichert, H. Vennekate, and R. Xiang, Simulation of ELBE SRF gun II for high-bunch-charge applications, Nucl. Instrum. Methods Phys. Res., Sect. A 830, 536 (2016).

[34] M. Krasilnikov et al., Electron beam asymmetry compensation with gun quadrupoles at PITZ, in Proceedings of the 2017 Free Electron Laser Conference, Santa Fe, NM (JACoW, Geneva, 2017), p. 421.

[35] D. H. Dowell, P. R. Bolton, J. E. Clendenin, S. M. Gierman, C. G. Limborg, B. F. Murphy, J. F. Schmerge, and T. Shaftan, Longitudinal emittance measurements at the SLAC gun test facility, Nucl. Instrum. Methods Phys. Res., Sect. A 507, 331 (2003).

[36] J. Teichert et al., Pulsed mode operation and longitudinal parameter measurement of the Rossendorf SRF gun, in Proceedings of the 2nd International Particle Accelerator Conference, San Sebastian, Spain (EPS-AG, Spain, 2011), p. 262.

[37] G. P. Williams, Filling the THz gap-High power sources and applications, Rep. Prog. Phys. 69, 301 (2006).

[38] H. A. Hafez, X. Chai, A. Ibrahim, S. Mondal, D. Férachou, $\mathrm{X}$. Ropagnol, and T. Ozaki, Intense terahertz radiation and their applications, J. Opt. 18, 093004 (2016).

[39] U. Lehnert, A. Aksoy, M. Helm, P. Michel, H. Schneider, W. Seidel, D. Stehr, and S. Winnerl, Conceptual design of a $\mathrm{THz}$ facility at the ELBE radiation source, in Proceedings of the 32nd Free Electron Laser Conference, Malmö, Sweden (Max-lab, Sweden, 2010), p. 656.

[40] P. Evtushenko, J. Coleman, K. Jordan, J. M. Klopf, G. Neil, and G. P. Williams, Bunch length measurements at JLab FEL, in Proceedings of the 2006 Free Electron Laser Conference, Berlin, Germany (JACoW, Geneva, 2006), p. 736. 